\title{
ELEMENTARY SUBGROUPS OF ISOTROPIC REDUCTIVE GROUPS
}

\author{
V. PETROV AND A. STAVROVA
}

\begin{abstract}
Let $G$ be a not necessarily split reductive group scheme over a commutative ring $R$ with 1 . Given a parabolic subgroup $P$ of $G$, the elementary group $\mathrm{E}_{P}(R)$ is defined to be the subgroup of $G(R)$ generated by $\mathrm{U}_{P}(R)$ and $\mathrm{U}_{P^{-}}(R)$, where $\mathrm{U}_{P}$ and $\mathrm{U}_{P^{-}}$are the unipotent radicals of $P$ and its opposite $P^{-}$, respectively. It is proved that if $G$ contains a Zariski locally split torus of rank 2, then the group $\mathrm{E}_{P}(R)=\mathrm{E}(R)$ does not depend on $P$, and, in particular, is normal in $G(R)$.
\end{abstract}

\section{§1. INTRODUCTION}

Let $G$ be a reductive algebraic group over a commutative ring $R$ with identity. Our aim is to give a definition of an elementary subgroup $\mathrm{E}(R)$ of the group of points $G(R)$, generalizing the notion of the elementary subgroup of a split reductive group and other similar concepts, and to show that under some natural restrictions, $\mathrm{E}(R)$ is normal in $G(R)$.

The notion of the elementary subgroup $\mathrm{E}_{n}(R)$ of the general linear group $\mathrm{GL}_{n}(R)$ was introduced by Bass [7] (while before it had been used implicitly by Whitehead in the study of homotopy types of CW-complexes) and served as a basis for his construction of algebraic $K$-theory. In particular, the nonstable $K_{1}$-functor is defined as the quotient $\mathrm{GL}_{n}(R) / \mathrm{E}_{n}(R)$, and $K_{2}$ as the kernel of a certain central extension of $\mathrm{E}_{n}(R)$. The definition of the elementary subgroup involves a fixed basis in $R^{n}$, but by the Suslin theorem [26], if $R$ is commutative and $n \geq 3$, then $\mathrm{E}_{n}(R)$ does not depend on the choice of a basis, or, in other words, is normal in $\mathrm{GL}_{n}(R)$. Various approaches to this result were discussed, for example, in [25, 35].

Later on, the elementary subgroup was defined for arbitrary split semisimple groups over $R$ as the subgroup generated by all elementary root unipotents $x_{\alpha}(\xi)$ or, what is the same, by the $R$-points of the unipotent radical of a Borel subgroup $B$ in $G$ and of the unipotent radical of the opposite Borel subgroup $B^{-}$(see, e.g., [1, 21]). In the same way as in the case of $G=\mathrm{GL}_{n}$, it turns out that when the ranks of all irreducible components of the root system of $G$ are at least 2, the elementary subgroup does not depend on the choice of a Borel subgroup, i.e., is normal in $G(R)$. For the orthogonal and symplectic groups, this fact was proved by Suslin and Kopeiko [27, 18, and by Fu An Li [19, and for arbitrary Chevalley groups by Abe [1] in the case of local rings and by Taddei [29] in the general case (cf. [2]). A simpler proof was given by Hazrat and Vavilov in [15]. The normality of the elementary subgroup in twisted Chevalley groups was proved by Suzuki 28, and by Bak and Vavilov [5].

2000 Mathematics Subject Classification. Primary 20G35.

Key words and phrases. Reductive group scheme, elementary subgroup, Whitehead group, parabolic subgroup. $3251)$.

The first author was supported by PIMS Postdoctoral Fellowship and by INTAS (grant no. 03-51- 
For classical groups, there are versions of the definition of the elementary subgroup that involve an involution and a "form parameter" (in the sense of Bak). In that case normality was proved by Vaserstein and Hong You [34, and by Bak and Vavilov [6]; see also the paper of the first author on the case of "odd" unitary groups [22]. Certainly, not all classical groups in the sense of Bak can be presented as groups of points of reductive group schemes, but as for the methods, these works are direct generalizations of those mentioned above.

For nonsplit almost simple groups over a field $k$, the following analog of the elementary subgroup, introduced by Tits [30], is often considered. Namely, Tits defined the group $G^{+}(k)$ (originally $G_{k}^{0}$ ) as the subgroup generated by the $k$-points of the unipotent radicals of all parabolic subgroups in $G$ defined over $k$. This definition is usually preferred, because it makes normality obvious, but in fact $G^{+}(k)$ is generated by the points of any two opposite unipotent radicals; see [10, Proposition 6.2]. Note that $G^{+}(k)$ is projectively simple in almost all cases [30, and the description of normal subgroups in $G(k)$ is reduced to the study of the so-called Whitehead group $G(k) / G^{+}(k)$, which is a natural analog of the $K_{1}$-functor. The famous Kneser-Tits problem asks whether the quotient is trivial in the case of a simply connected group $G$. It has an affirmative solution for number fields (the last step was recently done by Gille [14]), but in general the answer is negative even for groups of type $A_{l}$ (the Platonov counterexample; see [14, 23]).

For nonsplit classical groups over rings, Vaserstein [32, 33] defined the elementary subgroup as the subgroup generated by all Eichler-Siegel-Dickson transvections. Normality is again obvious, but Vaserstein showed that the elementary subgroup is generated by transvections of a certain kind. Essentially, he fixed a parabolic subgroup of type $P_{1}$ and considered points of its unipotent radical and of the unipotent radical of an opposite parabolic subgroup.

Finally, we mention another definition of an elementary group that arises in the Jordan theory [3, 20]. The elementary group corresponding to a Jordan or Kantor pair is the group generated by all "exponents" of its elements taken in the adjoint representation. Morally, these are subgroups of suitable adjoint semisimple groups generated by points of two opposed unipotent radicals of nilpotency class 1 or 2 .

This naturally leads us to the following definition generalizing all the definitions mentioned above.

Let $P$ be a parabolic subgroup of a reductive group $G$ over $R$, and let $\mathrm{U}_{P}$ be its unipotent radical. Since the base $\operatorname{Spec} R$ is affine, the group $P$ has a Levi subgroup $\mathrm{L}_{P}$ (see [12, Exp. XXVI, Cor. 2.3]). There is a unique parabolic subgroup $P^{-}$in $G$ that is opposite to $P$ with respect to $\mathrm{L}_{P}$ (that is, $P^{-} \cap P=\mathrm{L}_{P}$; see [12, Exp. XXVI, Th. 4.3.2]).

We define the elementary subgroup $\mathrm{E}_{P}(R)$ corresponding to $P$ as the subgroup of $G(R)$ generated as an abstract group by $\mathrm{U}_{P}(R)$ and $\mathrm{U}_{P^{-}}(R)$.

Note that if $\mathrm{L}_{P}^{\prime}$ is another Levi subgroup of $P$, then $\mathrm{L}_{P}^{\prime}$ and $\mathrm{L}_{P}$ are conjugate by some element $u \in \mathrm{U}_{P}(R)$ [12, Exp. XXVI, Cor. 1.8]; hence $\mathrm{E}_{P}(R)$ does not depend on the choice of a Levi subgroup or, respectively, of an opposite subgroup $P^{-}$. We shall show that, under some natural restrictions, $\mathrm{E}_{P}(R)$ does not depend on the choice of $P$ as well, and, in particular, is normal in $G(R)$.

Recall that the main invariant of a split reductive group $G$ over an algebraically closed field (as well as over a commutative ring; see [12, Exp. XXII]) is its root system $\Phi$ with respect to a split maximal torus $T$. Every parabolic subgroup $P$ of a split group is characterized up to conjugacy by its type $J \subseteq \Pi$, where $\Pi$ is a system of simple roots in $\Phi$. A classical way to generalize these notions to the case of a nonsplit reductive group over an arbitrary field $k$ (or over a local ring; see [12, Exp. XXVI, §7]) is to replace the 
root system $\Phi$ by the relative root system ${ }_{k} \Phi$ in the sense of Borel and Tits [9, 31] and to adjust appropriately the definition of the type of a parabolic subgroup (cf. \$2).

We return to the case of an arbitrary reductive group $G$ over a $\operatorname{ring} R$. Let $G^{a d}$ denote the corresponding adjoint algebraic group. We say that a parabolic subgroup $P$ in $G$ is strictly proper if for every maximal ideal $M$ in $R$ the image of $P_{R_{M}}$ in $G_{i}$ under the projection map is a proper subgroup in $G_{i}$, where $G_{R_{M}}^{a d}=\prod_{i} G_{i}$ is the decomposition of the semisimple group $G_{R_{M}}^{a d}$ into a product of simple groups. In the language of Borel and Tits (and of [12, Exp. XXVI, §7]) this condition can be restated as follows: the type of the parabolic subgroup $P_{R_{M}}$ meets every irreducible component of the relative root system of $G_{R_{M}}$.

Our main result is the following.

Theorem 1. Let $G$ be a reductive algebraic group over a commutative ring $R$. Assume that for any maximal ideal $M$ in $R$ all irreducible components of the relative root system of $G_{R_{M}}$ are of rank at least 2. Then $\mathrm{E}_{P}(R)$ does not depend on the choice of a strictly proper parabolic subgroup $P$. In particular, $\mathrm{E}(R)=\mathrm{E}_{P}(R)$ is normal in $G(R)$.

Remark 1. The condition that the ranks of irreducible components of the relative root system of $G_{R_{M}}$ are at least 2 is equivalent to the existence of split tori of rank at least 2 in every simple factor of the adjoint group $G_{R_{M}}^{a d}$.

Remark 2. In essence, the theorem says that if $P$ and $P^{\prime}$ are strictly proper parabolic subgroups in $G$, then $\mathrm{E}_{P^{\prime}}(R)=\mathrm{E}_{P}(R)$ in the following cases:

- when $P$ and $P^{\prime}$ are (locally) conjugate;

- when $P \leq P^{\prime}$ are comparable with respect to inclusion.

In the second case the condition on the ranks of irreducible components may be omitted (Lemma 12).

The key point in the proof of Theorem 1 is to apply an analog of the Quillen-Suslin lemma (Lemma 17), which essentially reduces the problem to the case of a local ring $R$. A $K_{0}$-analog of that lemma appeared in Quillen's solution of the Serre problem [24, while a $K_{1}$-version that we use was proposed by Suslin [26]. Over a local ring the assertion of the theorem remains true even without the restriction on the rank of the relative root system; it is readily implied by the local conjugacy of minimal parabolic subgroups ([12, Exp. XXVI, §5]).

Our main technical tool is relative root subschemes of $G$. In $\S \$ 3$ 4 we define the system of relative roots $\Phi_{P}$ of $G$ with respect to a parabolic subgroup $P$, generalizing the classical definition of the relative roots by Borel and Tits 9 mentioned above. Unlike the classical case, now $\Phi_{P}$ is not necessarily a root system. Next, by using faithfully flat descent, for any relative root $A \in \Phi_{P}$, we construct ( $\$$ Theorem 2) a projective $R$-module $V_{A}$ and a closed embedding of schemes (but not of group schemes in general)

$$
X_{A}: \mathrm{W}\left(V_{A}\right) \rightarrow G,
$$

where $\mathrm{W}\left(V_{A}\right)$ is the affine group scheme corresponding to $V_{A}$. The elements $X_{A}(v)$, $A \in \Phi_{P}, v \in V_{A}$, of $G(R)$ play the same role as elementary root unipotents in split groups. In particular, they generate $\mathrm{E}_{P}(R)$ and are subject to certain commutator relations that generalize Chevalley commutator formulas:

$$
\left[X_{A}(v), X_{B}(u)\right]=\prod_{i, j>0} X_{i A+j B}\left(N_{A B i j}(v, u)\right),
$$

where $N_{A B i j}: V_{A} \times V_{B} \rightarrow V_{i A+j B}$ are certain polynomial maps homogeneous of degree $i$ in the first argument and of degree $j$ in the second argument (Lemmas 9, 10). Under certain 
restrictions, these maps $N_{A B i j}$ are surjective, which corresponds to the invertibility of coefficients in the split case.

The authors are heartily grateful to Nikolai Vavilov for his encouraging attention to their work.

\section{§2. LOCAL ÉPINGLAGES AND PARABOLIC SUBGROUPS}

Let $G$ be a reductive algebraic group over a commutative ring $R$ with 1 . Recall that an épinglage (12, Exp. XXIII, Déf. 1.1]) $\mathcal{E}$ of $G$ consists of the following data:

- the root datum $\mathcal{R}=\left(\mathrm{X}, \mathrm{X}^{\vee}, \Phi, \Phi^{\vee}, \Pi\right)$ of $G$, including a fixed set of simple roots $\Pi \subseteq \Phi$

- a split maximal subtorus $T$ of $G$, together with an isomorphism $\mathrm{X}^{*}(T) \simeq \mathrm{X}$, where $\mathrm{X}^{*}(T)$ is the character lattice of $T$;

- for any $\alpha \in \Pi$, an isomorphism $x_{\alpha}: \mathbf{G}_{a} \stackrel{\sim}{\longrightarrow} X_{\alpha}$ between the additive group $\mathbf{G}_{a}$ and the corresponding root subgroup $X_{\alpha}$ of $G$, such that $T$ acts on $X_{\alpha}$ by means of $\alpha$.

Any épinglage can be extended to a Chevalley system, that is, a system of isomorphisms $x_{\alpha}$ for all $\alpha \in \Phi$, satisfying the Steinberg relations (in particular, the Chevalley commutator formulas). A reductive group $G$ is split if and only if it admits an épinglage.

For any two épinglages $\mathcal{E}$ and $\mathcal{E}^{\prime}$ of $G$, there exists a unique inner automorphism $\iota$ of $G$ that takes, locally in the fpqc-topology, one épinglage into another. More precisely, this means that $\iota$ takes $T$ to $T^{\prime}$, and there is an fpqc-covering

$$
\coprod \operatorname{Spec} S_{\mu} \rightarrow \operatorname{Spec} R
$$

together with certain elements $g_{\mu} \in G\left(S_{\mu}\right)$ and root data isomorphisms $\gamma_{\mu}: \mathcal{R} \rightarrow \mathcal{R}^{\prime}$ (we require that $\gamma_{\mu}(\Pi)=\Pi^{\prime}$ ) such that over any $S_{\mu}$ the morphism $\iota$ is the conjugation by $g_{\mu}$, the isomorphism

$$
\mathrm{X}_{S_{\mu}}^{\prime} \simeq \mathrm{X}^{*}\left(T_{\mu}^{\prime}\right) \simeq \mathrm{X}^{*}\left(T_{\mu}\right) \simeq \mathrm{X}_{S_{\mu}}
$$

induced by $\iota$ coincides with $\gamma_{\mu}^{-1}$, and $\iota \circ x_{\alpha}=x_{\gamma_{\mu}(\alpha)}^{\prime}$ for all $\alpha \in \Pi$ (see Exp. XXIV, Lemme 1.5). Observe that if Spec $S_{\mu}$ and Spec $S_{\nu}$ have nontrivial intersection (i.e., $S_{\mu} \otimes_{R} S_{\nu} \neq 0$ ), then $\gamma_{\mu}=\gamma_{\nu}$. Therefore, the entire collection of isomorphisms $\left\{\gamma_{\mu}\right\}$ does not depend on a given covering; we shall call these isomorphisms the patching symmetries between $\mathcal{E}$ and $\mathcal{E}^{\prime}$.

Let $P$ be a parabolic subgroup ([12, Exp. XXVI, Déf. 1.1]) of a split reductive group $G$. An épinglage $\mathcal{E}$ is said to be adapted to $P$ if there exists a parabolic set of roots $\Psi, \Pi \subseteq \Psi \subseteq \Phi$, such that $P$ is (algebraically) generated by the torus $T$ and the root subgroups $\bar{X}_{\gamma}, \gamma \in \Psi$. In particular, this implies that the unipotent radical $\mathrm{U}_{P}$ of $P$ is generated by $X_{\alpha}, \alpha \in \Psi \backslash-\Psi$. If a Levi subgroup $\mathrm{L}_{P}$ of $P$ is chosen, the épinglage is said to be adapted to $P$ and $\mathrm{L}_{P}$ if $\mathrm{L}_{P}$ is generated by $T$ and $X_{\alpha}, \alpha \in \Psi \cap-\Psi$, i.e., if $\mathrm{L}_{P}$ is a unique Levi subgroup of $P$ containing $T$ (see [12, Exp. XXVI, Prop. 1.12]).

If $\mathcal{E}$ and $\mathcal{E}^{\prime}$ are two épinglages of $G$ adapted to $P$, then the corresponding elements $g_{\mu}$ belong to $P\left(S_{\mu}\right)$ ([12, Exp. XXVI, Prop. 1.15]). If, moreover, these épinglages are adapted to a Levi subgroup $\mathrm{L}_{P}$ of $P$, then each $g_{\mu}$ belongs to $\mathrm{L}_{P}\left(S_{\mu}\right)$ (12, Exp. XXVI, Cor. 1.8]).

Assume now that $G$ is an arbitrary (i.e., not necessarily split) reductive algebraic group. Then $G$ is split locally in the étale, and hence also in the fpqc-topology ([12, Exp. XXII, Cor. 2.3]).

Let $P$ be a parabolic subgroup of $G$. Then fpqc-locally on $\operatorname{Spec} R$, one can choose an épinglage of $G$ adapted to $P$ ([12, Exp. XXVI, Lemme 1.14]). Since $G$ is a reductive 
group over an affine scheme $\operatorname{Spec} R, P$ possesses a Levi subgroup $\mathrm{L}_{P}$ (12, Exp. XXVI, Cor. 2.3]). Since, locally in the étale topology, $\mathrm{L}_{P}$ contains a maximal torus of $G$, we conclude that fpqc-locally one can choose an épinglage of $G$ adapted to both $P$ and $\mathrm{L}_{P}$.

By a local épinglage of $G$ we mean a triple $\tau$ consisting of

- an affine open subset $U_{\tau} \subseteq \operatorname{Spec} R$;

- its faithfully flat affine covering $\operatorname{Spec} S_{\tau} \rightarrow U_{\tau}$ such that $G$ splits over $S_{\tau}$;

- an épinglage $\mathcal{E}_{\tau}$ of $G_{S_{\tau}}$.

Consider the category whose objects are local épinglages $\tau$ and whose morphisms are graph isomorphisms between the Dynkin diagrams $D_{\tau}$ arising in the épinglages $\mathcal{E}_{\tau}$. This category is the symmetric groupoid $\operatorname{Sym}\left(\left\{D_{\tau}\right\}\right)$ determined by all Dynkin diagrams of all local épinglages $\tau$.

Two objects $\tau$ and $\sigma$ of $\operatorname{Sym}\left(\left\{D_{\tau}\right\}\right)$ provide two épinglages of the group $G_{S_{\tau} \otimes S_{\sigma}}$. Hence, they induce patching symmetries from $\mathcal{R}_{\tau}$ to $\mathcal{R}_{\sigma}$, which in turn give rise to certain graph isomorphisms between the corresponding Dynkin diagrams $D_{\tau}$ and $D_{\sigma}$. Define the patching groupoid $\Gamma$ to be the subgroupoid of $\operatorname{Sym}\left(\left\{D_{\tau}\right\}\right)$ generated by all these isomorphisms. We denote by $\Gamma_{\tau}$ the group of automorphisms of an object $\tau$ of $\Gamma$. Clearly, $\Gamma_{\tau}$ is a subgroup of the group of automorphisms Aut $\left(D_{\tau}\right)$. For example, if $R=k$ is a field and $S_{\tau}=K$ is a Galois extension, then $\Gamma_{\tau}$ coincides with the image of the Galois group $\operatorname{Gal}(K / k)$ in $\operatorname{Aut}\left(D_{\tau}\right)$ corresponding to the $*$-action of $\operatorname{Gal}(K / k)$ [31].

Consider a $\Gamma$-isomorphism class $\xi$ of local épinglages and denote by $U_{\xi}$ the union of all $U_{\tau}, \tau \in \xi$. It is easy to see that the open subsets $U_{\xi}$ form a partition of Spec $R$; in particular, they are clopen affine subschemes. Since $\operatorname{Spec} R$ is quasicompact, their number is finite. Therefore, we can write $U_{\xi}=\operatorname{Spec} R_{\xi}$, where $R \simeq \prod_{\xi} R_{\xi}$.

Note that for any parabolic subgroup $P$ of $G$, our definition of $\mathrm{E}_{P}(R)$ readily implies $\mathrm{E}_{P}(R) \simeq \prod_{\xi} \mathrm{E}_{P_{R_{\xi}}}\left(R_{\xi}\right)$. This allows us to reduce most questions on the elementary subgroup to the case where the groupoid $\Gamma$ consists of a unique isomorphism class.

Now we define the type of a parabolic subgroup $P$. First, consider all local épinglages $\tau$ adapted to $P$. Every subgroup $P_{S_{\tau}}$ is a standard parabolic subgroup corresponding to a set $J_{\tau} \subseteq \Pi_{\tau}$ of simple roots, so that $P_{S_{\tau}}$ is generated by the respective torus $T_{\tau}$ and by the root subgroups corresponding to the roots in whose decomposition the simple roots from $J_{\tau}$ appear with nonnegative coefficients. The collection of all $J_{\tau}$ is invariant under the morphisms of $\Gamma$ (in particular, every $J_{\tau}$ is invariant under $\Gamma_{\tau}$ ). It is easy to see that, starting with this data, in any local épinglage $\tau$ we can choose a subset $J_{\tau} \subseteq \Pi_{\tau}$ so that $\Gamma$-invariance still occurs. The total collection $\left\{J_{\tau}\right\}$ will be called the type of the parabolic subgroup $P$.

In fact, the constant schemes $D_{\tau}$ over $S_{\tau}$ can be glued together along the patching isomorphisms to produce a twisted constant scheme over $R$, which is called the Dynkin scheme of $G$ (see [12, Exp. XXIV, §3)]). In a similar way, their clopen subschemes $J_{\tau}$ can be glued to give a clopen subscheme of the Dynkin scheme, which is precisely what is called the type of a parabolic subgroup in [12. However, we prefer to keep to the above set-theoretic notions.

Recall that if $R$ is a local ring, there exists a unique maximal (with respect to inclusion) parabolic subgroup type, which comes from a minimal parabolic subgroup (12, Exp. XXVI, Cor. 5.7]).

\section{$\S 3$. Relative Roots}

Throughout this section, $\Phi$ is a reduced root system in an $l$-dimensional Euclidean space with the scalar product ( , ). We fix a set of simple roots $\Pi=\left\{\alpha_{1}, \ldots, \alpha_{l}\right\}$ in $\Phi$ (when $\Phi$ is irreducible, our numbering follows [1]), and we identify the elements of $\Pi$ with the corresponding vertices of the Dynkin diagram $D$ of $\Phi$. 
Fix a subset $J \subseteq \Pi$, and let $\Delta$ be the subsystem of $\Phi$ spanned by $\Pi \backslash J$. Any root $\alpha \in \Phi$ has a unique decomposition of the form

$$
\alpha=\sum_{1 \leq r \leq l} m_{r}(\alpha) \alpha_{r} .
$$

We set

$$
\alpha_{J}=\sum_{\substack{1 \leq r \leq l, \alpha_{r} \in J}} m_{r}(\alpha) \alpha_{r} .
$$

We call a linear combination $a$ of the elements of $J$ a shape (cf. [4) if there exists a root $\alpha \in \Phi \backslash \Delta$ with $\alpha_{J}=a$. In this case we also say that $\alpha$ is a root of shape $\alpha_{J}$.

Lemma 1. Take $\alpha, \beta, \gamma \in \Phi$ such that none of them is opposite to another and $\alpha+\beta+\gamma$ is a root. Then at least two of the sums $\alpha+\beta, \alpha+\gamma, \beta+\gamma$ are roots.

Proof. We can assume that $\Phi$ is irreducible. Set $\delta=\alpha+\beta+\gamma$. Since $(\delta, \alpha+\beta+\gamma)=$ $(\delta, \delta)>0$, one of the products $(\delta, \alpha),(\delta, \beta),(\delta, \gamma)$ is positive; let it be $(\delta, \alpha)$. Then $\delta-\alpha=\beta+\gamma$ is a root. Next, if $(\alpha, \beta+\gamma)<0$, then one of the products $(\alpha, \beta)$, $(\alpha, \gamma)$ is negative, and hence either $\alpha+\beta$ or $\alpha+\gamma$ is a root. If $(\alpha, \beta+\gamma) \geq 0$, then $(\delta, \beta+\gamma)=(\alpha, \beta+\gamma)+(\beta+\gamma, \beta+\gamma)>0$, which implies that one of $(\delta, \beta),(\delta, \gamma)$ is positive; that is, $\delta-\beta$ or $\delta-\gamma$ is a root.

Lemma 2. Suppose that $a, b, c$ are shapes and that $a+b=c$. Then for any root $\gamma$ of shape $c$ there exist roots $\alpha$ of shape $a$ and $\beta$ of shape $b$ such that $\alpha+\beta=\gamma$.

Proof. The relation $a+b=c$ implies that the shapes $a, b, c$ are linear combinations of simple roots from the same irreducible component of $\Phi$, so we assume that $\Phi$ is irreducible. We can represent $\gamma$ as a sum $\gamma=\alpha_{0}+\beta_{0}+\lambda_{1}+\cdots+\lambda_{n}$, where $\alpha_{0}$ is a root of shape $a, \beta_{0}$ is a root of shape $b$, and $\lambda_{i} \in \Delta$. We proceed by induction on $n$. The case where $n=0$ is obvious. If $\left(\gamma, \alpha_{0}\right)>0$ or $\left(\gamma, \beta_{0}\right)>0$, then $\gamma-\alpha_{0}$ or, respectively, $\gamma-\beta_{0}$ is a root; therefore, we can take $\alpha=\alpha_{0}, \beta=\gamma-\alpha_{0}$ or $\beta=\beta_{0}, \alpha=\gamma-\beta_{0}$. Otherwise $(\gamma, \gamma)>0$ implies that there exists $i$ such that $\left(\gamma, \lambda_{i}\right)>0$; that is, $\gamma^{\prime}=\gamma-\lambda_{i}$ is a root. By the inductive hypothesis we have $\gamma^{\prime}=\alpha^{\prime}+\beta^{\prime}$, where $\alpha^{\prime}$ is a root of shape $a$, and $\beta^{\prime}$ is a root of shape $b$. It remains to note that by Lemma 1, one of $\alpha^{\prime}+\lambda_{i}, \beta^{\prime}+\lambda_{i}$ is a root.

Let $\Gamma$ be a subgroup of $\operatorname{Aut}(D)$, and suppose that $J \subseteq \Pi$ is invariant under the action of $\Gamma$. Let $\Gamma$ act trivially on $\mathbb{Z}$. Then the Abelian group $\operatorname{Map}_{\Gamma}(J, \mathbb{Z})$ of all $\Gamma$-invariant maps from $J$ to $\mathbb{Z}$ is free, and its rank is equal to the number of $\Gamma$-orbits in $J$. We define a linear map

$$
\pi=\pi_{J, \Gamma}: \mathbb{Z} \Phi \rightarrow \operatorname{Map}_{\Gamma}(J, \mathbb{Z}),
$$

where $\mathbb{Z} \Phi$ is the root lattice, as follows: for $v=\sum_{\alpha_{i} \in \Pi} m_{i}(v) \alpha_{i} \in \mathbb{Z} \Phi$, we set

$$
\pi(v)\left(\alpha_{j}\right)=\sum_{\alpha_{i} \in \Gamma\left(\alpha_{j}\right)} m_{i}(v) \text { for any } \alpha_{j} \in \Pi .
$$

The set $\pi(\Phi) \backslash\{0\}$ will be called the set, or the system, of relative roots and will be denoted by $\Phi_{J, \Gamma}$. The rank rank $\Phi_{J, \Gamma}$ of a system of relative roots $\Phi_{J, \Gamma}$ is the rank of the group $\operatorname{Map}_{\Gamma}(J, \mathbb{Z})$.

Note that if $R$ is a local ring, $\Phi$ is the root system of a reductive algebraic group $G, J$ is the type of a minimal parabolic subgroup of $G$, and $\Gamma$ is the group of automorphisms of any object of the patching groupoid, then $\Phi_{J, \Gamma}$ is indeed a root system (maybe a nonreduced one, i.e., of type $B C_{l}$ ). If the group $G$ is semisimple, the rank of this root system is equal to the rank of a maximal split subtorus of $G$. See [12, Exp. XXVI, §7] or $[9, \S 5]$ for the details. In general, however, $\Phi_{J, \Gamma}$ is not a root system. 
It is clear that any relative root $A \in \Phi_{J, \Gamma}$ can be represented as a (unique) linear combination of relative roots from $\pi(\Pi)$. By the level $\operatorname{lev}(A)$ of a relative root $A$ we mean the sum of the coefficients in this decomposition.

We say that $A \in \Phi_{J, \Gamma}$ is a positive (respectively, negative) relative root if it is a nonnegative (respectively, nonpositive) linear combination of the elements of $\pi(\Pi)$. The sets of positive and negative relative roots will be denoted by $\Phi_{J, \Gamma}^{+}$and $\Phi_{J, \Gamma}^{-}$, respectively. It is seen immediately that $A \in \Phi_{J, \Gamma}^{ \pm}$if and only if $\pi^{-1}(A) \subseteq \Phi^{ \pm}$, and, in particular, $\Phi_{J, \Gamma}=\Phi_{J, \Gamma}^{+} \cup \Phi_{J, \Gamma}^{-}$.

Observe that the group of automorphisms $\Gamma$ acts on the set of irreducible components of the root system $\Phi$. If this action is transitive, the system of relative roots $\Phi_{J, \Gamma}$ is said to be irreducible. Clearly, any system of relative roots $\Phi_{J, \Gamma}$ is a disjoint union of irreducible ones; we call them the irreducible components of $\Phi_{J, \Gamma}$.

Clearly, for $\alpha_{i}, \alpha_{j} \in J$ we have $\pi\left(\alpha_{i}\right)=\pi\left(\alpha_{j}\right)$ if and only if $\alpha_{i}$ and $\alpha_{j}$ are in the same $\Gamma$-orbit. Moreover, $\left.\pi\right|_{\Delta}=0$; that is, $\pi(\alpha)=\pi\left(\alpha_{J}\right)$ for any root $\alpha$. If the group $\Gamma$ is trivial, then the relative roots are in one-to-one correspondence with the shapes defined by $J$.

Lemma 3. Let $\alpha, \beta \in \Phi$. Then $\pi(\alpha)=\pi(\beta)$ if and only if there exists $\sigma \in \Gamma$ such that $\sigma\left(\alpha_{J}\right)=\beta_{J}$.

Proof. The case where $\Gamma=\{\mathrm{id}\}$ is clear. It is also easily seen that we can assume $\Phi$ is irreducible. Moreover, we can replace the subset $J$ by any subset $J^{\prime} \subseteq \Pi$ that differs from $J$ by a union of one-element $\Gamma$-orbits. Then if $\Phi=D_{l}, l \geq 4$, everything reduces to the case where $J$ consists of a unique orbit, and the claim is obvious. This leaves us with the cases where $\Phi=A_{l}, l \geq 1$, and $\Phi=E_{6}$.

It is easily seen that if $\Phi=A_{l}$, then the shapes with respect to a $\Gamma$-invariant subset $J$ are in one-to-one correspondence with the roots of some root system $\Phi^{\prime}=A_{m}, m \leq l$, so that the action of $\Gamma$ coincides with the action of $\operatorname{Aut}\left(D^{\prime}\right)$, where $D^{\prime}$ is the Dynkin diagram of $\Phi^{\prime}$. Hence, we can assume $J=\Pi$. Then $\Phi_{J, \Gamma}$ can be identified with the relative root system ${ }_{k} \Phi$ of a quasi-split algebraic group of type ${ }^{2} A_{m}$ (defined over some field $k$ ) in the sense of Borel and Tits, and we can use the general theory of reductive groups over fields 9]. Namely, applying an element of the relative Weyl group ${ }_{k} W$, we pass to the case where $\pi(\alpha)=\pi(\beta)$ is a simple root of ${ }_{k} \Phi$, and the statement is clear.

Similarly, if $\Phi=E_{6}$ and $J \supseteq\left\{\alpha_{1}, \alpha_{6}\right\} \cup\left\{\alpha_{3}, \alpha_{5}\right\}$ contains two nontrivial $\Gamma$-orbits, we can assume that $J=\Pi$ and view $\Phi_{J, \Gamma}$ as a relative root system in the sense of Borel and Tits. But if $J$ consists of a unique nontrivial $\Gamma$-orbit, that is, if $J=\left\{\alpha_{1}, \alpha_{6}\right\}$ or $J=\left\{\alpha_{3}, \alpha_{5}\right\}$, our statement is obvious.

Lemma 4. Suppose $A, B, C \in \Phi_{J, \Gamma}$ and $A+B=C$. Then for any $\gamma \in \pi^{-1}(C)$ there exist $\alpha \in \pi^{-1}(A)$ and $\beta \in \pi^{-1}(B)$ such that $\alpha+\beta=\gamma$.

Proof. If $\Gamma$ is trivial, then relative roots coincide with shapes with respect to $J$, and our statement is merely Lemma 2, In general, Lemma 2 implies that it suffices to find shapes $a, b, c$ such that $\pi(a)=A, \pi(b)=B, \pi(c)=C$, and $a+b=c$. Next, transferring some of the roots $A, B, C$ to the other side of the identity $A+B=C$, we may assume that $A, B, C \in \Phi_{J, \Gamma}^{+}$. As in the proof of Lemma 3, we are reduced to the situation where $\Phi$ is irreducible and $J$ contains no one-element $\Gamma$-orbit. Then the case of $\Phi=D_{l}, l \geq 4$, is straightforward. To settle the other cases, let $\sigma$ denote a unique nontrivial element of $\Gamma$.

If $\Phi=A_{l}$, again as in the proof of Lemma 3, we can assume that $J=\Pi$, and the system of relative roots is a relative root system ${ }_{k} \Phi$ in the sense of Borel and Tits, corresponding to a quasi-split algebraic group of type ${ }^{2} A_{l}$ over a field $k$, and $\Gamma$ depicts the $*$-action of a Galois group 9 , 31. Since we can leave out any one-element orbit, it 
suffices to consider the case where $l=2 n$ is even. Then $\Phi_{J, \Gamma}=B C_{n}$. It is known 9 that any element of the Weyl group of $\Phi_{J, \Gamma}$ ("the relative Weyl group") can be lifted to an element of the Weyl group of $\Phi$, so we can assume that one of the relative roots $A, B$, say, $A$, is a simple root of $\Phi_{J, \Gamma}$, that is, $A=\pi\left(\alpha_{i}\right)$, or a multiple of a short simple root, that is, $A=\pi\left(\alpha_{n}+\alpha_{n+1}\right)$. Take some $\alpha \in \pi^{-1}(B), \gamma \in \pi^{-1}(C)$, and set $J^{\prime}=\Pi \backslash \pi^{-1}(A)$. Then $\alpha_{J^{\prime}}=\gamma_{J^{\prime}}$; hence by Lemma 3 we may assume that $\alpha_{J^{\prime}}=\gamma_{J^{\prime}}$. Now it is easy to show that $\pi(\alpha)+A=\pi(\gamma)$ implies $\alpha+\alpha_{i}=\gamma$ or $\alpha+\sigma\left(\alpha_{i}\right)=\gamma$ in the first case, and $\alpha+\alpha_{n}+\alpha_{n+1}=\gamma$ in the second.

Now, let $\Phi=E_{6}$, and let roots $\alpha, \beta, \gamma \in \Phi^{+}$be such that $\pi(\alpha)=A, \pi(\beta)=B$ and $\pi(\gamma)=C$. If the shapes $\alpha_{J}, \beta_{J}, \gamma_{J}$ have no coefficients greater than 1 , the problem reduces to the case where $\Phi=A_{5}$, discussed above. Otherwise $\alpha_{3}, \alpha_{5} \in J$ and we may suppose that $m_{3}(\gamma)=2$ without loss of generality. Then $m_{5}(\gamma)=2$ or $m_{5}(\gamma)=1$. If $J=\left\{\alpha_{3}, \alpha_{5}\right\}$, then the proof is finished by applying $\sigma$ to one of $\alpha_{J}, \beta_{J}$, so we consider $J=\left\{\alpha_{1}, \alpha_{3}, \alpha_{5}, \alpha_{6}\right\}$.

The case of $J=\left\{\alpha_{3}, \alpha_{5}\right\}$ being settled, we can assume that $\alpha_{J^{\prime}}+\beta_{J^{\prime}}=\gamma_{J^{\prime}}$, where $J^{\prime}=\left\{\alpha_{3}, \alpha_{5}\right\}$. If $m_{5}(\gamma)=2$, then $m_{1}(\gamma)=m_{6}(\gamma)=1$. If one of the roots $\alpha$, $\beta$ has a coefficient $\geq 2$, then, without loss of generality, $m_{3}(\alpha)=2$ and $m_{5}(\alpha)=1$, which implies $m_{1}(\alpha)=1$ and $m_{3}(\beta)=0, m_{5}(\beta)=1$. Then $m_{1}(\beta)=0$ and $m_{6}(\alpha)+m_{6}(\beta)=m_{6}(\gamma)$, so that $\alpha_{J}+\beta_{J}=\gamma_{J}$. If $m_{3}(\alpha)=m_{5}(\alpha)=m_{3}(\beta)=m_{5}(\beta)=1$, we can use the case of $J=\left\{\alpha_{1}, \alpha_{6}\right\}$. The case where $m_{3}(\gamma)=2, m_{5}(\gamma)=1$ is completely similar.

Lemma 5. If a relative root $A \in \Phi_{J, \Gamma}$ is contained in an irreducible component of rank at least 2 , then there exist noncollinear relative roots $B, C \in \Phi_{J, \Gamma}$ such that $A=B+C$. If $\Phi=G_{2}$, then $B$ and $C$ can be chosen so that $B-C \notin \Phi_{J, \Gamma}$.

Proof. We can assume $\Phi$ is irreducible. First, consider the case where $\Phi=G_{2}$. Since $\operatorname{rank} \Phi_{J, \Gamma} \geq 2$, in this case $\Phi=\Phi_{J, \Gamma}$ and the relative roots coincide with the usual ones. Since the Weyl group transfers any root into a simple one, we can assume that $A$ is a simple root of $G_{2}$. Then we take $B=\alpha_{1}+\alpha_{2}, C=-\alpha_{2}$ if $A=\alpha_{1}$ is short, and $B=3 \alpha_{1}+2 \alpha_{2}, C=-\left(3 \alpha_{1}+\alpha_{2}\right)$ if $A=\alpha_{2}$ is long.

Now, let $\Phi \neq G_{2}$. We can assume that $A$ is a positive relative root, i.e., $\pi^{-1}(A) \subseteq \Phi^{+}$.

First, suppose that $A=k \pi\left(\alpha_{r}\right)$, where $\alpha_{r} \in \Pi$ is a simple root, $k>0$. Let $\alpha_{s} \in J$ be a simple root that does not belong to the $\Gamma$-orbit of $\alpha_{r}$ and is the nearest to $\alpha_{r}$ on the Dynkin diagram among elements with this property. It is easily seen that for any $\alpha \in \pi^{-1}(A)$ there exists $\beta \in \pi^{-1}\left(\pi\left(\alpha_{s}\right)\right)$ such that $(\alpha, \beta)<0$ and thus $\alpha+\beta \in \Phi$. Indeed, for any $\alpha \in \pi^{-1}(A)$ we have $m_{s}(\alpha)=0$ by the definition of $\pi$, so we can take $\beta$ to be the sum of simple roots constituting the chain between $\alpha_{s}$ and the nearest simple root that appears in the decomposition of $\alpha$ with a nonzero coefficient. Now we can take $B=\pi(\alpha+\beta)$ and $C=\pi(-\beta)$. Since $\pi(\alpha)=k \pi\left(\alpha_{r}\right)$ and $\pi(-\beta)=-\pi\left(\alpha_{s}\right)$, the relative roots $B$ and $C$ are noncollinear.

Now, let $A \neq k \pi\left(\alpha_{r}\right)$. For any root $\alpha \in \pi^{-1}(A)$ there are $\operatorname{roots} \beta_{1}, \ldots, \beta_{n} \in \Pi$ such that $\alpha=\beta_{1}+\cdots+\beta_{n}$ and for any $1 \leq i \leq n$ the $\operatorname{sum} \beta_{1}+\cdots+\beta_{i}$ is a root. Let $i$ be the smallest possible index satisfying $\beta_{i+1}, \ldots, \beta_{n} \in \Delta$. Then $\beta_{i} \in J$ and $\pi\left(\beta_{1}+\cdots+\beta_{i-1}+\beta_{i}\right)=A$. Set $B=\pi\left(\beta_{1}+\cdots+\beta_{i-1}\right)$ and $C=\pi\left(\beta_{i}\right)$. The relative roots $B$ and $C$ are noncollinear, because otherwise we would have $A=k \pi\left(\beta_{i}\right)$ for some $k>0$.

\section{§4. Relative Root subschemes}

Throughout this section, we assume that the patching groupoid consists of a unique isomorphism class; $P$ is a fixed parabolic subgroup of $G$ of type $\left\{J_{\tau}\right\}$. Then the maps $\pi_{\tau}: \mathrm{X}^{*}\left(T_{\tau}\right) \rightarrow \operatorname{Map}_{\Gamma}\left(J_{\tau}, \mathbb{Z}\right)$ are transformed into each other by patching symmetries, 
so we can identify the corresponding systems of relative roots $\Phi_{J_{\tau}, \Gamma_{\tau}}$. We denote the resulting system by $\Phi_{P}$.

Let $\Psi \subseteq \Phi_{P}$ be a unipotent closed set of relative roots, that is, a subset that contains the sum of any two of its elements (if this sum is a relative root) and does not contain any collinear oppositely directed relative roots. Then the set $\pi^{-1}(\Psi)$ is a unipotent closed subset of $\Phi$ in the usual sense.

We fix a Levi subgroup $\mathrm{L}_{P}$ of $P$. It is clear that the local épinglages $\tau$ adapted to $P$ and $\mathrm{L}_{P}$ constitute an open covering of Spec $R$. For any such $\tau$ we define $\mathrm{U}_{\Psi, \tau}$ to be the subgroup of $G_{S_{\tau}}$ generated by all $X_{\alpha, \tau}, \alpha \in \pi^{-1}(\Psi)$. Since any two épinglages $\tau$ and $\sigma$ adapted to $\mathrm{L}_{P}$ are locally conjugate by an element of $\mathrm{L}_{P}$, patching symmetries take $\mathrm{U}_{\Psi, \tau}$ to $\mathrm{U}_{\Psi, \sigma}$. Hence, the groups $\mathrm{U}_{\Psi, \tau}$ glue together into a global subgroup $\mathrm{U}_{\Psi}$ of $G$.

In particular, in this way we obtain closed subgroups $\mathrm{U}_{(A)}$ of $G$, where $(A)$ is the set of all relative roots that are positive multiples of a relative root $A$. It is easily seen that $\mathrm{U}_{(i A)}$ is normal in $\mathrm{U}_{(A)}$ for any $i \geq 1$.

For any finitely generated projective $R$-module $V$, the functor $S \mapsto V \otimes_{R} S$ is represented by an affine group scheme $\mathrm{W}(V)=\operatorname{Spec} \operatorname{Sym}^{*}\left(V^{*}\right)$, where $V^{*}$ is the dual $R$-module and $\mathrm{Sym}^{*}$ is the symmetric algebra. A morphism of schemes $\mathrm{W}\left(V_{1}\right) \rightarrow \mathrm{W}\left(V_{2}\right)$ is then determined by an element of $\operatorname{Sym}^{*}\left(V_{1}^{*}\right) \otimes_{R} V_{2}$. If this element lies in $\operatorname{Sym}^{d}\left(V_{1}^{*}\right) \otimes_{R} V_{2}$, we say that the morphism is homogeneous of degree $d$. In particular, the morphisms of degree 1 are linear morphisms.

Theorem 2. For all relative roots $A \in \Phi_{P}$, there exist projective modules $V_{A}$ over $R$ and closed embeddings of schemes

$$
X_{A}: \mathrm{W}\left(V_{A}\right) \rightarrow G
$$

such that, over any local épinglage $\tau$ adapted to $P$ and $\mathrm{L}_{P}$, the modules $V_{A} \otimes_{R} S_{\tau}$ are free, and if a basis $e_{1}, \ldots, e_{k_{A}}$ of $V_{A} \otimes_{R} S_{\tau}$ is chosen, then the morphism $X_{A}$ is given by

$$
X_{A}\left(e_{1} a_{1}+\cdots+e_{k_{A}} a_{k_{A}}\right)=\prod_{j=1}^{k_{A}} x_{\gamma_{j}}\left(a_{j}\right) \cdot \prod_{i \geq 2} \prod_{\pi(\beta)=i A} x_{\beta}\left(p_{\beta, \tau}^{i}\left(a_{1}, \ldots, a_{k_{A}}\right)\right),
$$

where $\gamma_{j}, 1 \leq j \leq k_{A}$, are all roots of $\pi^{-1}(A)$, and each $p_{\beta, \tau}^{i}$ is a homogeneous polynomial of degree $i$.

These morphisms enjoy the following properties.

1) $X_{A}(0)=1$.

2) For any $g \in \mathrm{L}_{P}$, we have

$$
g X_{A}(v) g^{-1}=\prod_{i \geq 1} X_{i A}\left(\varphi_{g, A}^{i}(v)\right),
$$

where each $\varphi_{g, A}^{i}: \mathrm{W}\left(V_{A}\right) \rightarrow \mathrm{W}\left(V_{i A}\right)$ is homogeneous of degree $i$.

3) We have

$$
X_{A}(v) X_{A}(w)=X_{A}(v+w) \prod_{i>1} X_{i A}\left(q_{A}^{i}(v, w)\right),
$$

where each $q_{A}^{i}: \mathrm{W}\left(V_{A}\right) \times_{\text {Spec } R} \mathrm{~W}\left(V_{A}\right)=\mathrm{W}\left(V_{A} \oplus V_{A}\right) \rightarrow \mathrm{W}\left(V_{i A}\right)$ is homogeneous of degree $i$.

Proof. Over a local épinglage $\tau$, we define $V_{A, \tau}$ as $S_{\tau}^{k_{A}}$, where $k_{A}=\left|\pi^{-1}(A)\right|$. Consider the morphisms of schemes

$$
Y_{A, \tau}: \mathrm{W}\left(V_{A, \tau}\right) \rightarrow U_{A, \tau}
$$


given by

$$
Y_{A, \tau}\left(e_{1} a_{1}+\cdots+e_{k_{A}} a_{k_{A}}\right)=\prod_{j} x_{\gamma_{j}}\left(a_{j}\right),
$$

where the $\gamma_{j}$ are all roots of $\pi^{-1}(A)$ in some order. The Chevalley commutator formula shows that over Spec $S_{\tau}$ the morphisms $Y_{A, \tau}$ satisfy the analogs of properties 1 and 3, and also 2 for the elements of $\left(\mathrm{L}_{P}\right)_{\tau}$ belonging to the torus $T_{\tau}$ or to a root subgroup. Since over $S_{\tau}$ the big cell $\Omega_{\mathrm{L}_{P}}$ is dense in $\mathrm{L}_{P}$, property 2 holds true for all elements of $\left(\mathrm{L}_{P}\right)_{\tau}$.

Next, for any two local épinglages $\sigma, \tau$ we have

$$
\iota_{\sigma \tau}\left(Y_{A, \tau}(v)\right)=Y_{A, \sigma}\left(\varphi_{A, \sigma, \tau}(v)\right) \bmod \mathrm{U}_{(2 A)},
$$

where $\varphi_{A, \sigma, \tau}: V_{A, \tau} \otimes_{R} S_{\sigma} \rightarrow V_{A, \sigma} \otimes_{R} S_{\tau}$ are linear maps. Clearly, they satisfy the cocycle condition, and hence glue the modules $V_{A, \tau}$ into a projective $R$-module $V_{A}$. This means that there are linear isomorphisms $\theta_{A, \tau}: V_{A} \otimes_{R} S_{\tau} \rightarrow V_{A, \tau}$ such that $\varphi_{A, \sigma, \tau}=$ $\theta_{A, \sigma} \circ\left(\theta_{A, \tau}\right)^{-1}$.

Now we use induction on $j$ to construct sections $X_{A, \tau}^{j}: V_{A, \tau} \rightarrow \mathrm{U}_{A, \tau}$ such that

$$
\iota_{\sigma \tau}\left(X_{A, \tau}^{j}(v)\right)=X_{A, \sigma}^{j}\left(\varphi_{A, \sigma, \tau}(v)\right) \bmod \mathrm{U}_{((j+1) A)},
$$

where the $X_{A, \tau}^{j}$ are determined by (1). Then, for $j$ sufficiently large, since the morphisms $X_{A, \tau}^{j}$ are affine, they glue into a morphism $X_{A}: \mathrm{W}\left(V_{A}\right) \rightarrow \mathrm{U}_{(A)}$ defined globally, and all the properties we need follow by descent from the Chevalley commutator formula.

Set $X_{A, \tau}^{1}=Y_{A, \tau}$. Suppose we have already defined $X_{A, \tau}^{j}$. We are looking for a map $X_{A, \tau}^{j+1}$ of the form

$$
X_{A, \tau}^{j+1}(v)=X_{A, \tau}^{j}(v) Y_{(j+1) A, \tau}\left(\chi_{\tau}(v)\right)
$$

where $\chi_{\tau}: V_{A, \tau} \rightarrow V_{(j+1) A, \tau}$ is a homogeneous polynomial map. We also want it to satisfy the relation

$$
\begin{aligned}
& \iota_{\sigma \tau}\left(X_{A, \tau}^{j}(v) Y_{(j+1) A, \tau}\left(\chi_{\tau}(v)\right)\right) \\
& \quad=X_{A, \sigma}^{j}\left(\varphi_{A, \sigma, \tau}(v)\right) Y_{(j+1) A, \sigma}\left(\chi_{\sigma}\left(\varphi_{A, \sigma, \tau}(v)\right)\right) \bmod \mathrm{U}_{((j+2) A)},
\end{aligned}
$$

or equivalently,

$$
\begin{aligned}
& \iota_{\sigma \tau}\left(X_{A, \tau}^{j}(v)\right)^{-1} X_{A, \sigma}^{j}\left(\varphi_{A, \sigma, \tau}(v)\right) \\
& \quad=Y_{(j+1) A, \sigma}\left(\varphi_{(j+1) A, \sigma, \tau}\left(\chi_{\tau}(v)\right)-\chi_{\sigma}\left(\varphi_{A, \sigma, \tau}(v)\right)\right) \bmod \mathrm{U}_{((j+2) A)} .
\end{aligned}
$$

Let $\psi_{\sigma \tau}(v)$ denote a unique element of $V_{(j+1) A, \sigma} \otimes_{R} S_{\tau}$ satisfying

$$
\iota_{\sigma \tau}\left(X_{A, \tau}^{j}(v)\right)^{-1} X_{A, \sigma}^{j}\left(\varphi_{A, \sigma, \tau}(v)\right)=Y_{(j+1) A, \sigma}\left(\psi_{\sigma \tau}(v)\right) \bmod \mathrm{U}_{((j+2) A)} .
$$

Then routine computations give

$$
\psi_{\rho \tau}=\varphi_{(j+1) A, \rho, \sigma} \circ \psi_{\sigma \tau}+\psi_{\rho \sigma} \circ \varphi_{A, \sigma, \tau} .
$$

Taking $a_{\sigma \tau}=\theta_{(j+1) A, \sigma}^{-1} \circ \psi_{\sigma \tau} \circ \theta_{A, \tau}$, we rewrite this as

$$
a_{\rho \tau}=a_{\rho \sigma}+a_{\sigma \tau} .
$$

Our covering is acyclic with coefficients in $\mathrm{W}\left(V_{(j+1) A}\right)$, and $\mathrm{H}^{1}\left(\operatorname{Spec} R, \mathrm{~W}\left(V_{(j+1) A}\right)\right)=0$. Therefore, there exist functions $b_{\tau}$ such that

$$
a_{\sigma \tau}=b_{\tau}-b_{\sigma} .
$$


Now we set $\chi_{\tau}=\theta_{(j+1) A, \tau} \circ b_{\tau} \circ \theta_{A, \tau}^{-1}$, obtaining

$$
\psi_{\sigma \tau}=\varphi_{(j+1) A, \sigma, \tau} \circ \chi_{\tau}-\chi_{\sigma} \circ \varphi_{A, \sigma, \tau}
$$

as we wanted.

It remains to prove that the maps $\chi_{\tau}$ can be chosen so that they will be polynomial and homogeneous of degree $j+1$. The Chevalley commutator relations imply that this is so for $\psi_{\sigma \tau}$. We extend the base to the polynomial ring $R\left[Z_{1}, \ldots, Z_{k_{A}}\right]$ and set $v_{Z}=e_{1} Z_{1}+\cdots+e_{k_{A}} Z_{k_{A}} \in S_{\tau}\left[Z_{1}, \ldots, Z_{k_{A}}\right]^{k_{A}}$. We have $\chi_{\tau}\left(v_{Z}\right) \in S_{\tau}\left[Z_{1}, \ldots, Z_{k_{A}}\right]^{k_{(j+1) A}}$. We define $\chi_{\tau}^{\prime}\left(v_{Z}\right)$ to be the element of $S_{\tau}\left[Z_{1}, \ldots, Z_{k_{A}}\right]^{k_{(j+1) A}}$ whose $n$th component, $1 \leq n \leq k_{(j+1) A}$, is the homogeneous summand of the $n$th component of $\chi_{\tau}\left(v_{Z}\right)$ of degree $j+1$. Then for any $v=e_{1} a_{1}+\cdots+e_{k_{A}} a_{k_{A}}$ we define $\chi_{\tau}^{\prime}(v)$ to be the image of $\chi_{\tau}^{\prime}\left(v_{Z}\right)$ under the specialization homomorphism

$$
S_{\tau}\left[Z_{1}, \ldots, Z_{k_{A}}\right] \rightarrow S_{\tau}, \quad Z_{1} \mapsto a_{1}, \ldots, Z_{k_{A}} \mapsto a_{k_{A}} .
$$

It is easily seen that identity (2) remains true with $\chi_{\tau}^{\prime}$ instead of $\chi_{\tau}$. This finishes the proof.

Lemma 6. The map

$$
X_{\Psi}: \mathrm{W}\left(\bigoplus_{A \in \Psi} V_{A}\right) \rightarrow \mathrm{U}_{\Psi}, \quad\left(v_{A}\right)_{A} \mapsto \prod_{A} X_{A}\left(v_{A}\right),
$$

where the product is taken in any fixed order respecting the level, is an isomorphism of schemes.

Proof. The statement is verified easily over any local épinglage with the help of (1), and the general case follows by descent.

Lemma 7. For any $A \in \Psi$, let $f_{1}^{A}, \ldots, f_{n_{A}}^{A}, n_{A} \geq 1$, be a system of generators for $V_{A}$ over $R$. Then for any ring extension $R \rightarrow S$ the group of points $U_{\Psi}(S)$ is generated as an abstract group by the elements $X_{A}\left(\xi f_{i}^{A}\right), \xi \in S, A \in \Psi, 1 \leq i \leq n_{A}$.

Proof. This follows from item 3 in Theorem 2 and Lemma 6 .

From now on, we fix an ordering of the system of relative roots that respects the level. Then, for any unipotent closed set $\Psi \subseteq \Phi_{P}$, Lemma $[6$ allows us to define certain morphisms

$$
p_{\Psi, A}: \mathrm{U}_{\Psi} \rightarrow \mathrm{W}\left(V_{A}\right)
$$

("the coefficient" at the relative $\operatorname{root} A$ ).

Lemma 8. For any $g \in \mathrm{U}_{\Psi}(R)$ there exists $g(X) \in \mathrm{U}_{\Psi}(R[X])$ such that $g(0)=1$ and $g(1)=g$.

Proof. If $g=\prod_{A} X_{A}\left(v_{A}\right)$, we take $g(X)=\prod_{A} X_{A}\left(v_{A} X\right)$.

\section{$\S 5$. Chevalley commutator formulas}

We keep the assumption that the patching groupoid consists of a unique isomorphism class, and that $\mathrm{L}_{P}$ is a fixed Levi subgroup of the parabolic subgroup $P$.

For any relative roots $A, B \in \Phi_{P}$, we denote by $(A, B)$ the unipotent closed set of relative roots consisting of all linear combinations $i A+j B, i, j>0$, that are in $\Phi_{P}$.

Lemma 9. Let $A, B$ be relative roots satisfying $m A \neq-k B$ for any $m, k \geq 1$. Then the commutator subgroup $\left[X_{A}\left(V_{A}\right), X_{B}\left(V_{B}\right)\right]$ is contained in

$$
U_{(A, B)}=\prod_{i, j>0} X_{i A+j B}\left(V_{i A+j B}\right),
$$


and each map

$$
\begin{aligned}
N_{A B i j}: & V_{A} \times V_{B} \rightarrow V_{i A+j B}, \\
\left(v_{A}, v_{B}\right) & \mapsto p_{(A, B), i A+j B}\left(\left[X_{A}\left(v_{A}\right), X_{B}\left(v_{B}\right)\right]\right)
\end{aligned}
$$

is homogeneous of degrees $i$ and $j$ in the first and the second arguments, respectively.

Proof. This follows by descent from Theorem 2 and the Chevalley commutator formula in the split case.

Lemma 10. Assume that $A, B, A+B \in \Phi_{P}$ and $A, B$ are noncollinear.

1) If $A-B \notin \Phi_{P}$, then

$$
\operatorname{Im} N_{A B 11}=V_{A+B} .
$$

2) If $A-B \in \Phi_{P}$ and $\Phi \neq G_{2}$, then

$$
\operatorname{Im} N_{A B 11}+\operatorname{Im} N_{A-B, 2 B, 1,1}+\sum_{v \in V_{B}} \operatorname{Im}\left(N_{A-B, B, 1,2}(-, v)\right)=V_{A+B},
$$

where $\operatorname{Im} N_{A-B, 2 B, 1,1}=0$ if $2 B \notin \Phi_{P}$.

Proof. 1) By Lemma 4 the assumption implies that any $\gamma \in \pi^{-1}(A+B)$ decomposes as $\gamma=\alpha+\beta, \alpha \in \pi^{-1}(A), \beta \in \pi^{-1}(B)$, where $\alpha-\beta$ is not a root. Then the commutator $\left[X_{A}\left(e_{\alpha}\right), X_{B}\left(e_{\beta}\right)\right]$, taken modulo $U_{\Psi}$, where $\Psi=(A, B) \backslash\{A+B\}$, is of the form $x_{\alpha+\beta}( \pm 1)$ over any member $S_{\tau}$ of the covering. Hence, $\operatorname{Im}\left(N_{A B 11}\right)_{\tau}=V_{A+B} \otimes S_{\tau}$. Since $\operatorname{Im} N_{A B 11}$ is a submodule of $V_{A+B}$ defined over the base ring, we have $\operatorname{Im} N_{A B 11}=V_{A+B}$.

2 ) In the same way as in 1$)$, we deduce that over any $S_{\tau}$ the module $\operatorname{Im}\left(N_{A B 11}\right)_{\tau}$ contains $\pm e_{\gamma}$, where $\gamma$ is a short root in $\pi^{-1}(A+B)$. If $\gamma$ is a long root in $\pi^{-1}(A+B)$, our assumptions and Lemma 4 imply the existence of $\alpha \in \pi^{-1}(A-B)$ and $\beta \in \pi^{-1}(B)$ such that $\gamma=\alpha+2 \beta$. Using item 3 of Theorem 2 , we see that, over any $S_{\tau},\left[X_{A-B}\left(e_{\alpha}\right), X_{B}\left(e_{\beta}\right)\right]$ modulo $U_{\Psi}$, where $\Psi=(A-B, B) \backslash\{A+B\}$, equals

$$
x_{\alpha+2 \beta}( \pm 1) \cdot \prod_{\beta^{\prime} \in 2 B}\left[x_{\alpha}(1), x_{\beta^{\prime}}\left(u_{\beta^{\prime}}\right)\right], \quad u_{\beta^{\prime}} \in S_{\tau} .
$$

Consequently, $e_{\gamma} \in \operatorname{Im}\left(N_{A-B, B, 1,2}\right)_{\tau}\left(-, e_{\beta}\right)+\operatorname{Im}\left(N_{A-B, 2 B, 1,1}\right)_{\tau}$. We can represent $e_{\beta}$ as

$$
e_{\beta}=a_{1} f_{1}+\cdots+a_{m} f_{m}, \quad a_{i} \in S_{\tau}, \quad f_{i} \in V_{B} .
$$

It is easily seen that, for any $\delta \in \pi^{-1}(A-B)$,

$$
\begin{aligned}
{\left[X_{A-B}\left(e_{\delta}\right), X_{B}\left(e_{\beta}\right)\right]=} & {\left[X_{A-B}\left(e_{\delta}\right), \prod_{i} X_{B}\left(a_{i} f_{i}\right) \cdot X_{2 B}(w)\right] } \\
= & {\left[X_{A-B}\left(e_{\delta}\right), \prod_{i} X_{B}\left(a_{i} f_{i}\right)\right] \cdot\left[X_{A-B}\left(e_{\delta}\right), X_{2 B}(w)\right] } \\
= & \prod_{i}\left[X_{A-B}\left(e_{\delta}\right), X_{B}\left(a_{i} f_{i}\right)\right] \prod_{i<j}\left[X_{B}\left(a_{i} f_{i}\right),\left[X_{A-B}\left(e_{\delta}\right), X_{B}\left(a_{j} f_{j}\right)\right]\right] \\
& \cdot\left[X_{A-B}\left(e_{\delta}\right), X_{2 B}(w)\right] \bmod U_{\Psi}
\end{aligned}
$$

for some $w \in\left(V_{2 B}\right)_{\tau}$ (Theorem 2). Expanding commutators further, we see that $\left(N_{A-B, B, 1,2}\right)_{\tau}\left(e_{\delta}, e_{\beta}\right)$ lies in

$$
\begin{aligned}
\sum_{i}\left(N_{A-B, B, 1,2}\right)_{\tau} & \left(e_{\delta}, a_{i} f_{i}\right)+\operatorname{Im}\left(N_{A B 11}\right)_{\tau}+\operatorname{Im}\left(N_{A-B, 2 B, 1,1}\right)_{\tau} \\
& =\sum_{i}\left(N_{A-B, B, 1,2}\right)_{\tau}\left(a_{i}^{2} e_{\delta}, f_{i}\right)+\operatorname{Im}\left(N_{A B 11}\right)_{\tau}+\operatorname{Im}\left(N_{A-B, 2 B, 1,1}\right)_{\tau} .
\end{aligned}
$$


Summarizing, we see that $e_{\gamma}$ is in

$$
\sum_{i} \operatorname{Im}\left(N_{A-B, B, 1,2}\right)_{\tau}\left(-, f_{i}\right)+\operatorname{Im}\left(N_{A B 11}\right)_{\tau}+\operatorname{Im}\left(N_{A-B, 2 B, 1,1}\right)_{\tau},
$$

which finishes the proof.

Lemma 11. Suppose that $A \in \Phi_{P}$ lies in an irreducible component of rank at least 2. Then for any $v \in V_{A}$ there exist relative roots $B_{i}, C_{i} \in \Phi_{P}$ noncollinear to $A$, elements $v_{i} \in V_{B_{i}}, u_{i} \in V_{C_{i}}$, and integers $k_{i}, l_{i}>0, n_{i} \geq 0(1 \leq i \leq m)$ such that for any $\xi, \eta \in R$ we havel

$$
X_{A}\left(\xi \eta^{2} v\right)=\prod_{i=1}^{m} X_{B_{i}}\left(\xi^{k_{i}} \eta^{n_{i}} v_{i}\right)^{X_{C_{i}}\left(\eta^{l_{i}} u_{i}\right)}
$$

Proof. We view $\xi, \eta$ as free variables generating a polynomial ring $R[\xi, \eta]$, and work with $R[\xi, \eta]$-points of the functors $X_{A}, A \in \Phi_{P}$, instead of $R$-points. The statement is then obtained by specializing $\xi$ and $\eta$.

By Lemma 囵 there are noncollinear relative roots $B, C \in \Phi_{P}$ such that $A=B+C$, and $B-C$ is not a relative root if $\Phi=G_{2}$. Then, by Lemmas 9 and 10 (the commutator formulas are still available over the extension $R[\xi, \eta]$ of $R$ ), the element $X_{A}\left(\xi \eta^{2} v\right)$ is contained in the subgroup generated by

$$
\left[X_{B}\left(\xi \cdot V_{B}\right), X_{C}\left(\eta^{2} \cdot V_{C}\right)\right] \quad \text { and } \prod_{\substack{i, j>0, i, j) \neq(1,1)}} X_{i B+j C}\left(\xi^{i} \eta^{2 j} \cdot V_{i B+j C}\right),
$$

and also, if $B-C$ is a relative root, by

$$
\begin{array}{lc}
{\left[X_{B-C}\left(\xi \cdot V_{B-C}\right), X_{2 C}\left(\eta^{2} \cdot V_{2 C}\right)\right],} & \prod_{\substack{i, j>0,(i, j) \neq(1,1)}} X_{i(B-C)+2 j C}\left(\xi^{i} \eta^{2 j} \cdot V_{i(B-C)+2 j C}\right), \\
{\left[X_{B-C}\left(\xi \cdot V_{B-C}\right), X_{C}\left(\eta \cdot V_{C}\right)\right],} & \prod_{\substack{i, j>0,(i, j) \neq(1,2)}} X_{i(B-C)+j C}\left(\xi^{i} \eta^{j} \cdot V_{i(B-C)+j C}\right)
\end{array}
$$

and, by property 3 ) in Theorem 2 , by

$$
\prod_{i>1} X_{i A}\left(\xi^{i} \eta^{2 i} \cdot V_{i A}\right)
$$

Since $B$ and $C$ are noncollinear, all relative roots involved are either noncollinear to $A$, or have the form $i A, i>1$. Hence, we can use descending induction on $k=\operatorname{lev}(A)$.

Now let $P \leq P^{\prime}$ be two parabolic subgroups of $G$. Observe that if $\mathrm{L}_{P^{\prime}}$ is a Levi subgroup of $P^{\prime}$, then $P$ possesses a Levi subgroup $\mathrm{L}_{P}$ satisfying $\mathrm{L}_{P} \subseteq \mathrm{L}_{P^{\prime}}$ ([12, Exp. XXVI, Prop. 1.20]). Suppose that $\tau$ is a local épinglage adapted to $P$ and $\mathrm{L}_{P}$ and that $P$ is of type $J=J_{\tau} \subseteq \Pi_{\tau}=\Pi$. Since $P \subseteq P^{\prime}$, the local épinglage $\tau$ is a fortiori adapted to $P^{\prime}$ and $\mathrm{L}_{P^{\prime}}$ (cf. [12, Exp. XXVI, Prop. 1.4]), and the parabolic subgroup $P^{\prime}$ is of type $J^{\prime} \subseteq J$.

Lemma 12. Let $P \leq P^{\prime}$ be strictly proper parabolic subgroups of $G$. Then there exists $k>0$ depending only on $\operatorname{rank} \Phi_{P}$ such that for any relative root $A \in \Phi_{P}$ and any $v \in V_{A}$ there exist relative roots $B_{i}, C_{i j} \in \Phi_{P^{\prime}}$, elements $v_{i} \in V_{B_{i}}, u_{i j} \in V_{C_{i j}}$, and integers

\footnotetext{
${ }^{1}$ We use exponential notation for conjugation, with $x^{y}=y^{-1} x y$.
} 


$$
\begin{aligned}
k_{i}, n_{i}, l_{i j}>0\left(1 \leq i \leq m, 1 \leq j \leq m_{j}\right) \text { that satisfy } \\
X_{A}\left(\xi \eta^{k} v\right)=\prod_{i=1}^{m} X_{B_{i}}\left(\xi^{k_{i}} \eta^{n_{i}} v_{i}\right) \prod_{j=1}^{m_{i}} X_{C_{i j}}\left(\eta^{l_{i j}} u_{i j}\right) .
\end{aligned}
$$

for any $\xi, \eta \in R$. In particular, $\mathrm{E}_{P}(R)=\mathrm{E}_{P^{\prime}}(R)$.

Proof. Let $\Theta \subseteq \Phi^{+}$be the set of positive roots corresponding to the unipotent radical $\mathrm{U}_{P^{\prime}}$. Clearly, $-\Theta$ corresponds to the unipotent radical $\mathrm{U}_{\left(P^{\prime}\right)^{-}}$. Then in the notation of $\$ 4$ we have $\mathrm{U}_{P^{\prime}}=\mathrm{U}_{\Psi}, \mathrm{U}_{\left(P^{\prime}\right)^{-}}=\mathrm{U}_{-\Psi}$, where $\Psi=\pi(\Theta) \subseteq \Phi_{P}^{+}$is the corresponding set of relative roots.

Fix an order on $\Phi^{+}$in such a way that the induced order on $\Phi_{P^{\prime}}^{+}$respects the level. Without loss of generality, we take $A \in \Phi_{P}^{+}$. If $A \in \Psi$, then by Lemma 6 there are morphisms of schemes

$$
\lambda_{B}=p_{\Phi_{P^{\prime}}^{+}, B} \circ X_{A}: \mathrm{W}\left(V_{A}\right) \rightarrow \mathrm{W}\left(V_{B}\right), \quad B \in \Phi_{P^{\prime}}^{+},
$$

such that $X_{A}(u)=\prod_{B \in \Phi_{P^{\prime}}^{+}} X_{B}\left(\lambda_{B}(u)\right)$ for any $u \in V_{A}$, where the product is taken in the chosen order. The Chevalley commutator formulas and descent imply that the $\lambda_{B}$, $B \in \Phi_{P^{\prime}}^{+}$, are homogeneous polynomial maps. Hence, for any $A \in \Psi$ (and similarly, for any $A \in(-\Psi)$ ) the statement of the lemma holds true with $u_{i j}=0,1 \leq i, j \leq m$.

Now, consider the case where $A \notin \Psi$. The types $J$ and $J^{\prime}$ of $P$ and $P^{\prime}$ are both invariant under the group of automorphisms $\Gamma_{\tau}=\Gamma$, that is, are unions of some $\Gamma$-orbits of simple roots. Suppose first that $J \backslash J^{\prime}$ consists of a unique $\Gamma$-orbit containing a simple root $\alpha_{r} \in \Pi$. Then $\Psi=\Phi_{P}^{+} \backslash \mathbb{N} \pi\left(\alpha_{r}\right)$, so we can assume that $A \in \Phi_{P}^{+}$is of the form $A=n \pi\left(\alpha_{r}\right), n \in \mathbb{Z}$. Since $P^{\prime}$ is strictly proper, the rank of the irreducible component of $\Phi_{P}$ containing $A$ is at least 2. Then our statement readily follows from Lemma 11 (with $\xi$ replaced by $\xi \eta$ ) and the preceding case, because any root $B \in \Phi_{P}$ noncollinear to $A$ automatically belongs to $\Psi \cup(-\Psi)=\Phi_{P} \backslash \mathbb{Z} \pi\left(\alpha_{r}\right)$.

Now if $J \backslash J^{\prime}$ consists of more than one $\Gamma$-orbit, the proof is finished by induction, with the use of the fact that, for any $\Gamma$-invariant subset $J^{\prime \prime} \subseteq \Pi$ such that $J^{\prime} \subseteq J^{\prime \prime} \subseteq J$, there exists a (strictly proper) parabolic subgroup $P^{\prime \prime}$ of $G$ containing $P$ and having type $J^{\prime \prime}$ ([12, Exp. XXVI, Lemma 3.8]).

\section{§6. Quillen-Suslin lemma and the Proof of Theorem 1}

We introduce some additional notation. For an ideal $I$ of the ring $R$, we denote by $G(R, I)$ the kernel of the reduction homomorphism $G(R) \rightarrow G(R / I)$, by $\mathrm{U}_{\Psi}(R, I)$ the intersection $\mathrm{U}_{\Psi}(R) \cap G(R, I)$, by $\mathrm{E}_{P}(I)$ the subgroup generated by $\mathrm{U}_{P}(R, I)$ and $\mathrm{U}_{P^{-}}(R, I)$, and by $\mathrm{E}_{P}(R, I)$ the normal closure of $\mathrm{E}_{P}(I)$ in $\mathrm{E}_{P}(R)$. Also, for any maximal ideal $M$ of the ring $R$, we denote by $F_{M}$ the localization homomorphism $G(R) \rightarrow G\left(R_{M}\right)$.

Lemma 13. $\mathrm{E}_{P}(R[X]) \simeq \mathrm{E}_{P}(R[X], X R[X]) \rtimes \mathrm{E}_{P}(R)$.

Proof. The group $\mathrm{E}_{P}(R[X], X R[X])$ is normal in $\mathrm{E}_{P}(R[X])$ by definition, and its intersection with $\mathrm{E}_{P}(R)$ is trivial. So it suffices to prove that $\mathrm{U}_{P}(R)$ and $\mathrm{U}_{P}(R[X], X R[X])$ generate $\mathrm{U}_{P}(R[X])$. Obviously, we can assume that the patching groupoid consists of a unique isomorphism class. Then the statement follows from Lemma 7.

Corollary. $\mathrm{E}_{P}(R[X]) \cap G(R[X], X R[X])=\mathrm{E}_{P}(R[X], X R[X])$.

Proof. Take an element $g(X)$ of $\mathrm{E}_{P}(R[X]) \cap G(R[X], X R[X])$; it can be presented as $g_{1}(X) \cdot g_{2}$, where $g_{1}(X) \in \mathrm{E}_{P}(R[X], X R[X]), g_{2} \in \mathrm{E}_{P}(R)$. Then $g_{2}=g_{1}(0) \cdot g_{2}=$ $g(0)=1$. 
Lemma 14. Let $g(Z), h(Z) \in G(R[Z])$ be such that $F_{M}(g(Z))=F_{M}(h(Z))$ and $g(0)=$ $h(0)$. Then there exists $s \in R \backslash M$ such that $g(s Z)=h(s Z)$.

Proof. The corresponding statement for $\mathbb{A}^{n}$ is clear, and $G$ is a closed subscheme of $\mathbb{A}^{n}$ for some $n$.

From now on we assume the hypothesis of Theorem 1 .

Lemma 15. For any $g(Z) \in \mathrm{E}_{P}\left(R_{M}[Z], Z R_{M}[Z]\right)$ there exist $h(Z) \in \mathrm{E}_{P}(R[Z], Z R[Z])$ and $s \in R \backslash M$ such that $F_{M}(h(Z))=g(s Z)$.

Proof. We can assume that the patching groupoid of $G$ (over $R$ ) consists of a unique isomorphism class. Indeed, if the closed point $M$ of $\operatorname{Spec} R$ lies in an open subset $U_{\xi}=$ Spec $R_{\xi}$ (see \$2), and $h^{\prime}(Z) \in \mathrm{E}_{P}\left(R_{\xi}[Z], Z R_{\xi}[Z]\right)$ is an element mapped to $g(s Z)$, we can take an $h(Z)$ that is equal to $h^{\prime}(Z)$ over $U_{\xi}$ and to 1 over $\coprod_{\eta \neq \xi} U_{\eta}$.

The proof of Lemma 13 shows that $\mathrm{E}_{P}\left(R_{M}[Z]\right)$ is generated by $\mathrm{E}_{P}\left(Z R_{M}[Z]\right)$ and $\mathrm{E}_{P}\left(R_{M}\right)$. Hence it suffices to consider elements $g(Z)$ of the form $g_{1} g_{2}(Z) g_{1}^{-1}$, where $g_{1} \in \mathrm{E}_{P}\left(R_{M}\right)$ and $g_{2}(Z) \in \mathrm{E}_{P}\left(Z R_{M}[Z]\right)$. Set $S=R \backslash M$. It is easily seen that for any $s^{\prime} \in S$ there exists $s \in S$ such that $g_{2}(s Z)$ belongs to $F_{M}\left(\mathrm{E}_{P}\left(s^{\prime} Z R[Z]\right)\right)$. It remains to prove that there exists $s^{\prime} \in S$ satisfying

$$
g_{1} F_{M}\left(\mathrm{E}_{P}\left(s^{\prime} Z R[Z]\right)\right) g_{1}^{-1} \subseteq F_{M}\left(\mathrm{E}_{P}(R[Z], Z R[Z])\right) .
$$

Instead, we prove that for any $s^{\prime \prime} \in S$ there exists $s^{\prime} \in S$ such that

$$
g_{1} F_{M}\left(\mathrm{E}_{P}\left(s^{\prime} Z R[Z]\right)\right)^{F_{M}\left(\mathrm{E}_{P}\left(s^{\prime} R[Z]\right)\right)} g_{1}^{-1} \subseteq F_{M}\left(\mathrm{E}_{P}\left(s^{\prime \prime} Z R[Z]\right)\right)^{F_{M}\left(\mathrm{E}_{P}\left(s^{\prime \prime} R[Z]\right)\right)} .
$$

Then we can assume that $g_{1}$ is a root generator of $\mathrm{E}_{P}\left(R_{M}\right)$.

Let $P_{\text {min }}$ be the minimal parabolic subgroup of $G_{R_{M}}$ contained in $P_{R_{M}}$, and let $\Phi_{P_{\min }}$ be the corresponding system of relative roots. Lemma 12 implies that $\mathrm{E}_{P}\left(R_{M}\right)=$ $\mathrm{E}_{P_{R_{M}}}\left(R_{M}\right)$ coincides with $\mathrm{E}_{P_{\min }}\left(R_{M}\right)$, so we can take $g_{1}=X_{A}(v)$ for some $A \in \Phi_{P_{\min }}$, $v \in V_{A}$. Moreover, by Lemma 12, we have $X_{A}(t v) \in F_{M}\left(\mathrm{E}_{P}(R)\right)$ for some $t=t\left(g_{1}\right) \in S$.

By Lemma 7 the group $F_{M}\left(\mathrm{E}_{P}\left(s^{\prime} R[Z]\right)\right)$ (respectively, $\left.F_{M}\left(\mathrm{E}_{P}\left(s^{\prime} Z R[Z]\right)\right)\right)$ is generated by the elements $h_{0}$ of the form $X_{C}\left(\xi s^{\prime} F_{M}\left(e_{C, i}\right)\right)$ (respectively, $X_{C}\left(\xi s^{\prime} Z F_{M}\left(e_{C, i}\right)\right)$ ), where $C \in \Phi_{P}, \xi \in R[Z]$, and the elements $e_{C, i}$ span $V_{C} \otimes R[Z]$ over $R[Z]$. To prove (3), it suffices to show that $g_{1} h_{0} g_{1}^{-1} \in F_{M}\left(\mathrm{E}_{P}\left(s^{\prime \prime} R[Z]\right)\right)$ (respectively, $g_{1} h_{0} g_{1}^{-1} \in$ $\left.F_{M}\left(\mathrm{E}_{P}\left(s^{\prime \prime} Z R[Z]\right)\right)^{F_{M}\left(\mathrm{E}_{P}\left(s^{\prime \prime} R[Z]\right)\right)}\right)$ for all generators $h_{0}$ with $\xi=1$, because the general statement follows readily if we replace $Z$ by $\xi Z$.

Taking, in Lemma 12, $\xi=1, \eta=s^{\prime}$ (respectively, $\xi=Z, \eta=s^{\prime}$ ), and $s^{\prime}=\left(s^{\prime \prime \prime}\right)^{k}$ for some $s^{\prime \prime \prime} \in S$, we can represent $h_{0}$ as a (finite) product of elements $h$ of the form $X_{B}\left(s^{\prime \prime \prime} u\right)$ (respectively, $X_{B}\left(s^{\prime \prime \prime} Z u\right) \Pi_{i} X_{D_{i}}\left(s^{\prime \prime \prime} w_{i}\right)$ ), where $B \in \Phi_{P_{\min }}, u \in V_{B} \otimes R_{M}[Z]$, $D_{i} \in \Phi_{P_{\min }}, w_{i} \in V_{D_{i}} \otimes R_{M}[Z]$. Clearly, we can restrict ourselves to the elements $h$ of the form $X_{B}\left(s^{\prime \prime \prime} u\right)$ (respectively, $X_{B}\left(s^{\prime \prime \prime} Z u\right)$ ). As above, by Lemma 12 we have $h \in F_{M}\left(\mathrm{E}_{P}(R[Z])\right)$ (respectively, $\left.F_{M}\left(\mathrm{E}_{P}(Z R[Z])\right)\right)$ as soon as $s^{\prime \prime \prime}$ is divisible by a certain $r=r(h) \in S$.

Next, in the case where $m B \neq-k A$ for any $m, k \geq 1$, Lemma 9 obviously implies $g_{1} h g_{1}^{-1} \in F_{M}\left(\mathrm{E}_{P}\left(s^{\prime \prime} R[Z]\right)\right)$ (respectively, $\left.g_{1} h g_{1}^{-1} \in F_{M}\left(\mathrm{E}_{P}\left(s^{\prime \prime} Z R[Z]\right)\right)\right)$ if $s^{\prime \prime \prime}$ is divisible by $s^{\prime \prime}$ and by certain powers of $t$ and $r$. Consider the case where $A$ and $B$ are collinear.

By the assumption of Theorem 1, the rank of any irreducible component of $\Phi_{P_{\min }}$ is at least 2. Then, by Lemma [11, for any $u \in V_{B} \otimes R_{M}[Z]$ we can find relative roots $B_{1}, \ldots$, $B_{m}, C_{1}, \ldots, C_{m} \in \Phi_{P_{\min }}$ noncollinear to $B$ (and hence to $A$ ), elements $v_{i} \in V_{B_{i}} \otimes R_{M}[Z]$, $u_{i} \in V_{C_{i}} \otimes R_{M}[Z]$, and integers $k_{i}, l_{i}>0, n_{i} \geq 0(1 \leq i \leq m)$ such that

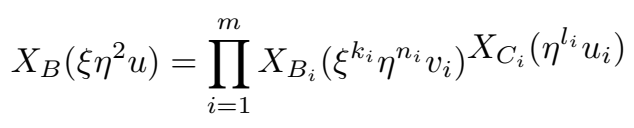


for any $\xi, \eta \in R_{M}[Z]$. Set $\xi=s_{1}$ (respectively, $Z s_{1}$ ), $\eta=s_{2}$, where both $s_{1}, s_{2} \in S$ are divisible by sufficiently large powers of $t$ and $s^{\prime \prime}$, and also by $p \in S$ such that $p u_{i}, p v_{i} \in$ $R[Z]$ for any $1 \leq i \leq m$. Now, if $s^{\prime \prime \prime} \in S$ is divisible by $s_{1} s_{2}^{2}$, then by Lemmas 9 and 12 we obtain

$$
g_{1} h g_{1}^{-1} \in F_{M}\left(\mathrm{E}_{P}\left(s^{\prime \prime} R[Z]\right)\right)^{F_{M}\left(\mathrm{E}_{P}\left(s^{\prime \prime} R[Z]\right)\right)}=F_{M}\left(\mathrm{E}_{P}\left(s^{\prime \prime} R[Z]\right)\right)
$$

(respectively,

as required.

$$
\left.g_{1} h g_{1}^{-1} \in F_{M}\left(\mathrm{E}_{P}\left(s^{\prime \prime} Z R[Z]\right)\right)^{F_{M}\left(\mathrm{E}_{P}\left(s^{\prime \prime} R[Z]\right)\right)}\right)
$$

Lemma 16. For any $g(X) \in G(R[X])$ such that $F_{M}(g(X))$ lies in $\mathrm{E}_{P}\left(R_{M}[X]\right)$, there exists $s \in R \backslash M$ satisfying $g(a X) g(b X)^{-1} \in \mathrm{E}_{P}(R[X])$ for all $a, b \in R$ such that $a \equiv b$ $\bmod s$.

Proof. Consider the element $f(Z)=g(X(Y+Z)) g(X Y)^{-1} \in G(R[X, Y, Z])$. Observe that $F_{M}(f(Z)) \in \mathrm{E}_{P}\left(R_{M}[X, Y, Z]\right)$ and $f(0)=1$. By the corollary to Lemma [13, $F_{M}(f(Z))$ belongs to $\mathrm{E}_{P}\left(R_{M}[X, Y, Z], Z R_{M}[X, Y, Z]\right)$. Now, by Lemma 15, there exists $h(Z) \in \mathrm{E}_{P}(R[X, Y, Z], Z R[X, Y, Z])$ and $s_{1} \notin M$ such that $F_{M}(h(Z))=F_{M}\left(f\left(s_{1} Z\right)\right)$. By Lemma 14, there is $s_{2} \notin M$ such that $h\left(s_{2} Z\right)=f\left(s_{1} s_{2} Z\right)$. Set $s=s_{1} s_{2}$; then $g(X(Y+s Z)) g(X Y)^{-1}$ lies in $\mathrm{E}_{P}(R[X, Y, Z])$. Now we specialize $Y$ and $Z$ to obtain the statement we need.

Lemma 17. Let $g(X) \in G(R[X])$ be such that $g(0) \in \mathrm{E}_{P}(R)$, and suppose $F_{M}(g(X)) \in$ $\mathrm{E}_{P}\left(R_{M}[X]\right)$ for all maximal ideals $M$. Then $g(X) \in \mathrm{E}_{P}(R[X])$.

Proof. For any maximal ideal $M$, we choose $s_{M} \notin M$ as in Lemma 16. Since the ideal generated by all $s_{M}$ 's is not contained in any maximal one, there is a partition of unity $1=\sum_{i=1}^{N} s_{M_{i}} t_{i}$. We apply the Abel method of summation by parts: if $a_{j}$ denotes the partial sum $\sum_{i=1}^{N-j} s_{M_{i}} t_{i}$, then $a_{j+1} \equiv a_{j} \bmod s_{M_{N-j}}$, and we have

$$
g(X)=\left(\prod_{j=0}^{N-1} g\left(a_{j} X\right) g\left(a_{j+1} X\right)^{-1}\right) g(0),
$$

where all factors are in $\mathrm{E}_{P}(R[X])$.

Proof of Theorem 1. Let $Q$ be a parabolic subgroup of $G$ distinct from $P$. Let $g \in$ $\mathrm{E}_{Q}(R)$; we need to prove that $g \in \mathrm{E}_{P}(R)$. We may assume that $g \in \mathrm{U}_{Q}(R)$. Choose $g(X) \in \mathrm{U}_{Q}(R[X])$ as in Lemma 8 , and let $M$ be a maximal ideal of $R$. By [12, Exp. XXVI, Cor. 5.2 and Cor. 5.7], over $R_{M}$ both parabolic subgroups $P$ and $Q$ contain some minimal parabolic subgroups $P_{\min }$ and $Q_{\min }$, and these subgroups are conjugate by an element $h \in \mathrm{E}_{P_{\min }}\left(R_{M}\right)$. Now, $F_{M}(g(X))$ lies in $\mathrm{U}_{Q}\left(R_{M}[X]\right)$, and a fortiori in $\mathrm{U}_{Q_{\min }}\left(R_{M}[X]\right)$. Hence, $h F_{M}(g(X)) h^{-1}$, and then also $F_{M}(g(X))$, are inside the group $\mathrm{E}_{P_{\min }}\left(R_{M}[X]\right)$, which coincides with $\mathrm{E}_{P}\left(R_{M}[X]\right)$ by Lemma 12. Since $g(0)=1$, Lemma 17implies that $g(X)$ is in $\mathrm{E}_{P}(R[X])$. But $g=g(1)$, so $g$ lies in $\mathrm{E}_{P}(R)$, and the theorem is proved.

\section{$\S 7$. EXAMPLES}

1. Let $D$ be an Azumaya algebra over $R$, of degree $d$. The group $G=\operatorname{GL}_{r+1}(D)$ is a reductive algebraic group of type $A_{(r+1) d-1}$ (more precisely, the functor $S \mapsto \mathrm{GL}_{r+1}(D \otimes S)$ is represented by a reductive group scheme $G$ ). The subgroup $P \leq G$ consisting of upper triangular matrices is a parabolic subgroup of type $\{d, 2 d, \ldots,(r+1) d\}$. The system $\Phi_{P}$ of relative roots with respect to $P$ is a root system of type $A_{r}$. The module $V_{A}$ corresponding to relative roots $A \in \Phi_{P}$ can be identified with $D$, so that the maps $N_{\varepsilon_{i}-\varepsilon_{j}, \varepsilon_{j}-\varepsilon_{k}, 11}: D \times D \rightarrow D$ coincide with multiplication in $D$. The elementary subgroup 
$\mathrm{E}_{P}(R)$ coincides with the subgroup $\mathrm{E}_{r+1}(D) \subseteq \mathrm{GL}_{r+1}(D)$ generated by elementary matrices.

2. Let $V$ be a projective module of rank $2 n$ endowed with a nondegenerate quadratic form $Q$. Consider the group $\mathrm{O}(V, Q)$ of $Q$-invariant automorphisms of $V$. One can define (see, e.g., [16]) the Dickson map from $\mathrm{O}(V, Q)$ to $(\mathbb{Z} / 2 \mathbb{Z})_{R}$; if $2 \in R^{*}$, this map coincides with the usual determinant map. Its kernel $\mathrm{O}^{+}(V, Q)$ is a reductive algebraic group of type $D_{n}$. Suppose that $V$ contains $r<n$ pairwise orthogonal hyperbolic pairs $\left(e_{1}, f_{1}\right), \ldots,\left(e_{r}, f_{r}\right)$ (i.e., $Q\left(e_{i}\right)=Q\left(f_{i}\right)=0$ and $\left.Q\left(e_{i}+f_{j}\right)=\delta_{i j}\right)$. Then the subgroup $P \leq \mathrm{O}^{+}(V, Q)$ of automorphisms that preserve the flag

$$
\left\langle e_{1}\right\rangle \leq\left\langle e_{1}, e_{2}\right\rangle \leq \cdots \leq\left\langle e_{1}, \ldots, e_{r}\right\rangle
$$

is a parabolic subgroup of type $\{1, \ldots, r\}$. The respective relative roots form a root system of type $B_{r}$. The module $V_{A}$ corresponding to a relative root $A$ can be identified with $R$ if $A$ is long, and with the orthogonal complement to $\left\langle e_{1}, \ldots, e_{r}, f_{1}, \ldots, f_{r}\right\rangle \subseteq V$ if $A$ is short. If $A, B$, and $A+B$ are relative roots, the map $N_{A B 11}$ looks like this:

- $(u, v) \mapsto \pm(Q(u+v)-Q(u)-Q(v))$ if $A$ and $B$ are short;

- $(a, b) \mapsto \pm a b$ if $A$ and $B$ are long;

- $(a, v) \mapsto \pm a v$ if $A$ is long and $B$ is short.

If $A$ is a long root and if $B$ is a short root such that $A+2 B$ is also a root, then the map $N_{A B 12}$ takes $(a, v) \in V_{A} \times V_{B}$ to $\pm a Q(v)$. The elementary subgroup $\mathrm{E}_{P}(R)$ coincides with the group generated by the so-called Eichler-Siegel-Dickson transvections.

3. Let $S$ be a quadratic étale extension of $R$, i.e., a twisted form of the algebra $R \times R$. Then $S$ possesses an involution $x \mapsto \bar{x}$ obtained by twisting the involution $(a, b) \mapsto(b, a)$. The set of ${ }^{-}$-stable elements of $S$ coincides with $R$. The map $\operatorname{tr}: S \rightarrow R, x \mapsto x+\bar{x}$, is called the trace map.

Let $V$ be a projective $S$-module of rank $n+1$ endowed with a nondegenerate form $H$. The group $\mathrm{U}(V, H)$ of $H$-invariant automorphisms of $V$ is a reductive group over $R$, of type ${ }^{2} A_{n}$ (index 2 means that the group is of outer type; that is, the automorphism group of an object of the patching groupoid consists of two elements). Suppose that $V$ contains $r \leq \frac{n}{2}$ pairwise orthogonal hyperbolic pairs $\left(e_{1}, f_{1}\right), \ldots,\left(e_{r}, f_{r}\right)$ (i.e., $H\left(e_{i}, e_{i}\right)=$ $H\left(f_{i}, f_{i}\right)=0$ and $\left.H\left(e_{i}, f_{j}\right)=\delta_{i j}\right)$. The subgroup $P \leq \mathrm{U}(V, H)$ of automorphisms that preserve the flag

$$
\left\langle e_{1}\right\rangle \leq\left\langle e_{1}, e_{2}\right\rangle \leq \cdots \leq\left\langle e_{1}, \ldots, e_{r}\right\rangle
$$

is a parabolic subgroup of type $\{1, \ldots, r, n-r+1, \ldots, n\}$. The relative roots form a root system of type $B C_{r}$. The module $V_{A}$ corresponding to a relative root $A$ can be identified with $S$ if $A$ is short, with the orthogonal complement to $\left\langle e_{1}, \ldots, e_{r}, f_{1}, \ldots, f_{r}\right\rangle \subseteq V$ if $A$ is extra short, and with $\operatorname{ker} \operatorname{tr}$ if $A$ is long. If $A, B$, and $A+B$ are relative roots, the map $N_{A B 11}$ looks like this:

- $(u, v) \mapsto \pm H(u, v)$ if $A$ and $B$ are extra short;

- $(a, b) \mapsto \pm a b$ if $A, B$ and $A+B$ are short;

- $(a, v) \mapsto \pm a v$ if $A$ is short, $B$ is extra short;

- $(a, b) \mapsto \pm(a b-\bar{b} \bar{a})$ if $A$ and $B$ are short, $A+B$ is long;

- $(a, b) \mapsto \pm a b$ if $A$ is long and $B$ is short;

- $(u, v) \mapsto \pm(H(u, v)-H(v, u))$ if $A=B$ is extra short.

If $A+2 B$ is a relative root, then the map $N_{A B 12}$ looks like this:

- $(a, b) \mapsto \pm \bar{b} a b$ if $A$ is long and $B$ is short;

- $(a, v) \mapsto \pm a \sigma(H(v, v))$, where $\sigma$ is a certain fixed section of tr, if $A$ is short and $B$ is extra short. 
4. Recall that an algebra is said be alternative if any two elements generate an associative subalgebra. A Cayley algebra over a $\operatorname{ring} R$ is an alternative algebra $C$ with 1 endowed with an involution $x \mapsto \bar{x}$ and such that $C$ is a projective $R$-module of constant rank 8 , and the norm map $n(x)=\bar{x} x=x \bar{x}$ takes values in $R$ and is a nondegenerate quadratic form on $C$. Then the trace map $t(x)=x+\bar{x}$ on $C$ also takes values in $R$.

Given a Cayley algebra $C$ and three invertible scalars $\gamma_{1}, \gamma_{2}, \gamma_{3} \in R$, we can construct the cubic Jordan algebra $J=\mathcal{H}_{3}\left(C, \gamma_{1}, \gamma_{2}, \gamma_{3}\right)$ consisting of the matrices

$$
\left(\begin{array}{ccc}
\xi_{1} & c_{3} & \gamma_{1}^{-1} \gamma_{3} \bar{c}_{2} \\
\gamma_{2}^{-1} \gamma_{1} \bar{c}_{3} & \xi_{2} & c_{1} \\
c_{2} & \gamma_{3}^{-1} \gamma_{2} \bar{c}_{1} & \xi_{3}
\end{array}\right)
$$

with $c_{1}, c_{2}, c_{3} \in C$ and $\xi_{1}, \xi_{2}, \xi_{3} \in R$. In particular, there is a norm on $J$, which is a cubic map $N: J \rightarrow R$; the norm of the matrix (4) equals

$$
\xi_{1} \xi_{2} \xi_{3}-\gamma_{3}^{-1} \gamma_{2} \xi_{1} n\left(c_{1}\right)-\gamma_{1}^{-1} \gamma_{3} \xi_{2} n\left(c_{2}\right)-\gamma_{2}^{-1} \gamma_{1} \xi_{1} n\left(c_{3}\right)+t\left(c_{1} c_{2} c_{3}\right) .
$$

See [17] or [8] for the details.

Set

$$
e_{1}=\left(\begin{array}{lll}
1 & 0 & 0 \\
0 & 0 & 0 \\
0 & 0 & 0
\end{array}\right), \quad e_{2}=\left(\begin{array}{lll}
0 & 0 & 0 \\
0 & 1 & 0 \\
0 & 0 & 0
\end{array}\right), \quad e_{3}=\left(\begin{array}{lll}
0 & 0 & 0 \\
0 & 0 & 0 \\
0 & 0 & 1
\end{array}\right)
$$

Observe that $e_{1}, e_{2}, e_{3}$ are pairwise orthogonal idempotents in $J$ with sum 1 . We denote by $c[i j]$, where $c \in C$ and $1 \leq i \neq j \leq 3$, the matrix of the form (44) with $\gamma_{j} c$ at the position $(i, j)$ and zeros at all positions distinct from $(i, j)$ and $(j, i)$.

The functor

$$
S \mapsto\left\{g \in \mathrm{GL}(J \otimes S) \mid N(g x)=N(x) \text { for all } x \in J \otimes S^{\prime}, S \subseteq S^{\prime}\right\}
$$

is represented by a semisimple group scheme $G$ of type $E_{6}$. The subgroup $P \leq G$ of automorphisms that preserve the flag

$$
\mathbf{0} \leq\left(\begin{array}{lll}
* & 0 & 0 \\
0 & 0 & 0 \\
0 & 0 & 0
\end{array}\right) \leq\left(\begin{array}{lll}
* & * & 0 \\
* & * & 0 \\
0 & 0 & 0
\end{array}\right) \leq J
$$

is a parabolic subgroup of type $\left\{\alpha_{1}, \alpha_{6}\right\}$ (cf. [13]). The first nontrivial member of the flag is spanned by $e_{1}$, and the second coincides with the summand $J_{0}\left(e_{3}\right)$ of the Pierce decomposition induced by $e_{3}$ (i.e., with the set of elements of $J$ cancelled by $e_{3}$ ).

The corresponding relative roots form a root system of type $A_{2}$, where $\Phi_{P}=$ $\left\{ \pm\left(\varepsilon_{1}-\varepsilon_{2}\right), \pm\left(\varepsilon_{2}-\varepsilon_{3}\right), \pm\left(\varepsilon_{1}-\varepsilon_{3}\right)\right\}$ in the notation of [11]. The module $V_{A}$ corresponding to a relative root $A$ can be identified with $C$. Then the element $X_{\varepsilon_{i}-\varepsilon_{j}}(c)$ is the "algebraic transvection" $T_{\gamma_{j}^{-1} c[i j], e_{j}}$, and the map $N_{\varepsilon_{i}-\varepsilon_{j}, \varepsilon_{j}-\varepsilon_{k}, 11}: C \times C \rightarrow C$ coincides with multiplication in $C$ (see, e.g., [8, (v)]). The elementary subgroup $\mathrm{E}_{P}(R)$ is the subgroup generated by all algebraic transvections.

\section{REFERENCES}

[1] E. Abe, Chevalley groups over local rings, Tôhoku Math. J. (2) 21 (1969), 474-494. MR0258837 $(41: 3483)$

[2] , Normal subgroups of Chevalley groups over commutative rings, Algebraic K-Theory and Algebraic Number Theory (Honolulu, HI, 1987), Contemp. Math., vol. 83, Amer. Math. Soc., Providence, RI, 1989, pp. 1-17. MR0991973 (91a:20046)

[3] B. N. Allison and J. R. Faulkner, Elementary groups and invertibility for Kantor pairs, Comm. Algebra 27 (1999), 519-556. MR1671930 (99k:17002)

[4] H. Azad, M. Barry, and G. Seitz, On the structure of parabolic subgroups, Comm. Algebra 18 (1990), 551-562. MR1047327 (91d:20048) 
[5] A. Bak and N. Vavilov, Normality for elementary subgroup functors, Math. Proc. Cambridge Philos. Soc. 118 (1995), 35-47. MR1329456 (96d:20046)

[6] Structure of hyperbolic unitary groups. I. Elementary subgroups, Algebra Colloq. 7 (2000), 159-196. MR1810843 (2002b:20070)

[7] H. Bass, K-theory and stable algebra, Inst. Hautes Études Sci. Publ. Math. No. 22 (1964), 5-60. MR0174604(30:4805)

[8] R. Bix, Octonion planes over local rings, Trans. Amer. Math. Soc. 261 (1980), 417-438. MR0580896 (81k:17013)

[9] A. Borel and J. Tits, Groupes réductifs, Inst. Hautes Études Sci. Publ. Math. No. 27 (1965), 55-150. MR0207712 (34:7527)

[10] _ Homomorphismes "abstraits" de groupes algébriques simples, Ann. of Math. (2) 97 (1973), 499-571. MR0316587 (47:5134)

[11] N. Bourbaki, Éléments de mathématique. Fasc. 34. Groupes et algèbres de Lie. Ch. 4-6, Actualités Sci. Indust., No. 1337, Hermann, Paris, 1968. MR0240238 (39:1590)

[12] M. Demazure and A. Grothendieck, Schémas en groupes, Lecture Notes in Math., vols. 151153, Springer-Verlag, Berlin, 1970. MR0274458 (43:223a) MR0274459 (43:223b) MR0274460 (43:223c)

[13] S. Garibaldi and M. Carr, Geometries, the principle of duality, and algebraic groups, Expo. Math. 24 (2006), 195-234. MR2250947 (2007f:20080)

[14] Ph. Gille, Le problème de Kneser-Tits, Sèm. Bourbaki 2007, Exp. 983, 1-39.

[15] R. Hazrat and N. Vavilov, $K_{1}$ of Chevalley groups are nilpotent, J. Pure Appl. Algebra 179 (2003), 99-116. MR1958377 (2004i:20081)

[16] M.-A. Knus, Quadratic and Hermitian forms over rings, Grundlehren Math. Wiss., Bd. 294, Springer-Verlag, Berlin, 1991. MR.1096299 (92i:11039)

[17] M.-A. Knus, A. Merkurjev, M. Rost, and J.-P. Tignol, The book of involutions, Amer. Math. Soc. Colloq. Publ., vol. 44, Amer. Math. Soc., Providence, RI, 1998. MR1632779 (2000a:16031)

[18] V. I. Kopeǐko, Stabilization of symplectic groups over a ring of polynomials, Mat. Sb. 106 (1978), no. 1, 94-107; English transl., Math. USSR-Sb. 34 (1978), no. 5, 655-669. MR0497932 (80f:13008)

[19] $\mathrm{Fu} \mathrm{An} \mathrm{Li}$, The structure of orthogonal groups over arbitrary commutative rings, Chinese Ann. Math. Ser. B 10 (1989), 341-350. MR1027673 (90k:20084)

[20] O. Loos, Elementary groups and stability for Jordan pairs, K-Theory 9 (1995), 77-116. MR.1340841 (96f:17038)

[21] H. Matsumoto, Subgroups of finite index in certain arithmetic groups, Algebraic Groups and Discontinuous Subgroups (Proc. Sympos. Pure Math., Boulder, Colo., 1965), Proc. Sympos. Pure Math., vol. 9, Amer. Math. Soc., Providence, RI, 1966, pp. 99-103. MR0204534 (34:4373)

[22] V. A. Petrov, Odd unitary groups, Zap. Nauchn. Sem. S.-Peterburg. Otdel. Mat. Inst. Steklov. (POMI) 305 (2003), 195-225; English transl., J. Math. Sci. (N.Y.) 130 (2005), no. 3, 4752-4766. MR:2033642 (2005b:20100)

[23] V. P. Platonov and A. S. Rapinchuk, Algebraic groups and number theory, "Nauka", Moscow, 1991; English transl., Pure Appl. Math., vol. 139, Acad. Press, Inc., Boston, MA, 1994. MR1137274 (93j:11023) MR:1278263 (95b:11039)

[24] D. Quillen, Projective modules over polynomial rings, Invent. Math. 36 (1976), 167-171. MR:0427303 (55:337)

[25] A. Stepanov and N. Vavilov, Decomposition of transvections: A theme with variations, $K$-Theory 19 (2000), 109-153. MR.1740757 (2000m:20076)

[26] A. A. Suslin, On the structure of the special linear group over polynomial rings, Izv. Akad. Nauk SSSR Ser. Mat. 41 (1977), no. 2, 235-252; English transl., Math. USSR-Izv. 11 (1977), 221-238. MR0472792(57:12482)

[27] A. A. Suslin and V. I. Kopeǐko, Quadratic modules and the orthogonal group over polynomial rings, Zap. Nauchn. Sem. Leningrad. Otdel. Mat. Inst. Steklov. (LOMI) 71 (1977), 216-250; English transl., J. Soviet Math. 20 (1982), no. 6, 2665-2691. MR0469914 (57:9694)

[28] K. Suzuki, Normality of the elementary subgroups of twisted Chevalley groups over commutative rings, J. Algebra 175 (1995), 526-536. MR1339654(96m:20077)

[29] G. Taddei, Normalité des groupes élémentaires dans les groupes de Chevalley sur un anneau, Applications of Algebraic K-Theory to Algebraic Geometry and Number Theory. Part II (Boulder, Colo., 1983), Contemp. Math., vol. 55, Amer. Math. Soc., Providence, RI, 1986, pp. 693-710. MR0862660 (88a:20054)

[30] J. Tits, Algebraic and abstract simple groups, Ann. of Math. (2) 80 (1964), 313-329. MR0164968 $(29: 2259)$ 
[31] _ Classification of algebraic semisimple groups, Algebraic Groups and Discontinuous Subgroups (Proc. Sympos. Pure Math., Boulder, Colo., 1965), Proc. Sympos. Pure Math., vol. 9, Amer. Math. Soc., Providence, RI, 1966, pp. 33-62. MR0224710 (37:309)

[32] L. N. Vaserstein, Normal subgroups of orthogonal groups over commutative rings, Amer. J. Math. 110 (1988), 955-973. MR0961501 (89i:20071)

[33] _ Normal subgroups of symplectic groups over rings, K-Theory 2 (1989), 647-673. MR0999398 (90f:20064)

[34] L. N. Vaserstein and Hong You, Normal subgroups of classical groups over rings, J. Pure Appl. Algebra 105 (1995), 93-105. MR1364152 (96k:20096)

[35] N. A. Vavilov, Structure of Chevalley groups over commutative rings, Nonassociative Algebras and Related Topics (Hiroshima, 1990), World Sci. Publ., River Edge, NJ, 1991, pp. 219-335. MR.1150262 (92k:20090)

University of Alberta, Edmonton, Canada

E-mail address: victorapetrov@googlemail.com

St. Petersburg State University, St. Petersburg, Russia

E-mail address: a_stavrova@mail.ru

Received 21/DEC/2007

Translated by THE AUTHORS 